\title{
S1P Lyase Regulation of Thymic Egress and Oncogenic Inflammatory Signaling
}

\author{
Ashok Kumar, ${ }^{1}$ Jesus Zamora-Pineda, ${ }^{2}$ Emilie Degagné, ${ }^{2}$ and Julie D. Saba ${ }^{2}$ \\ ${ }^{1}$ Department of Biochemistry, All India Institute of Medical Sciences (AIIMS) Bhopal, Madhya Pradesh 462020, India \\ ${ }^{2}$ Children's Hospital Oakland Research Institute and UCSF Benioff Children's Hospital Oakland, 5700 Martin Luther King Jr. Way, \\ Oakland, CA 94609, USA
}

Correspondence should be addressed to Julie D. Saba; jsaba@chori.org

Received 12 May 2017; Accepted 13 September 2017; Published 3 December 2017

Academic Editor: Alice Alessenko

Copyright (c) 2017 Ashok Kumar et al. This is an open access article distributed under the Creative Commons Attribution License, which permits unrestricted use, distribution, and reproduction in any medium, provided the original work is properly cited.

\begin{abstract}
Sphingosine-1-phosphate (S1P) is a potent lipid signaling molecule that regulates pleiotropic biological functions including cell migration, survival, angiogenesis, immune cell trafficking, inflammation, and carcinogenesis. It acts as a ligand for a family of cell surface receptors. S1P concentrations are high in blood and lymph but low in tissues, especially the thymus and lymphoid organs. S1P chemotactic gradients are essential for lymphocyte egress and other aspects of physiological cell trafficking. S1P is irreversibly degraded by S1P lyase (SPL). SPL regulates lymphocyte trafficking, inflammation and other physiological and pathological processes. For example, SPL located in thymic dendritic cells acts as a metabolic gatekeeper that controls the normal egress of mature $\mathrm{T}$ lymphocytes from the thymus into the circulation, whereas SPL deficiency in gut epithelial cells promotes colitis and colitis-associated carcinogenesis (CAC). Recently, we identified a complex syndrome comprised of nephrosis, adrenal insufficiency, and immunological defects caused by inherited mutations in human SGPL1, the gene encoding SPL. In the present article, we review current evidence supporting the role of SPL in thymic egress, inflammation, and cancer. Lastly, we summarize recent progress in understanding other SPL functions, its role in inherited disease, and SPL targeting for therapeutic purposes.
\end{abstract}

\section{Introduction}

Sphingosine-1-phosphate (S1P) is a sphingolipid metabolite and a potent signaling molecule that regulates diverse cellular functions such as cell proliferation, differentiation, and migration as well as complex processes including development, vascular maturation, angiogenesis, immune function, and inflammation [1-3]. S1P mediates most of its biological actions by binding to five S1P receptors (S1PRs) that belong to the family of $\mathrm{G}$ protein-coupled receptors [3]. Intracellular and circulatory S1P levels are tightly regulated by its synthesis and catabolism. S1P is generated by the phosphorylation of the long-chain base sphingosine, a step catalyzed by sphingosine kinase [4]. The resulting S1P product can be dephosphorylated by various lipid phosphatases $[5,6]$. In addition, S1P is irreversibly degraded by S1P lyase (SPL), which cleaves S1P at the C2-3 carbon-carbon bond, yielding two products, ethanolamine phosphate and the long-chain aldehyde trans-2-hexadecenal [7-9]. Cleavage of S1P by SPL represents the final step in the sphingolipid catabolic pathway. When this step is blocked, there is no alternative pathway for sphingolipid degradation to occur. SPL depletes S1P in cellular, vascular, and tissue compartments and regulates steadystate S1P levels. Thus, SPL controls S1P pools available for interactions with intracellular targets and for autocrine and paracrine receptor-mediated signaling [9]. SPL regulates many physiological and pathological phenomena including maintenance of pluripotency in embryonic stem cells, DNA damage response, chemoresistance, muscle regeneration, satellite cell activation, thymic egress, glomerular function, adrenal function, atherosclerosis, ischemia-reperfusion injury, experimental autoimmune encephalomyelitis (EAE), 


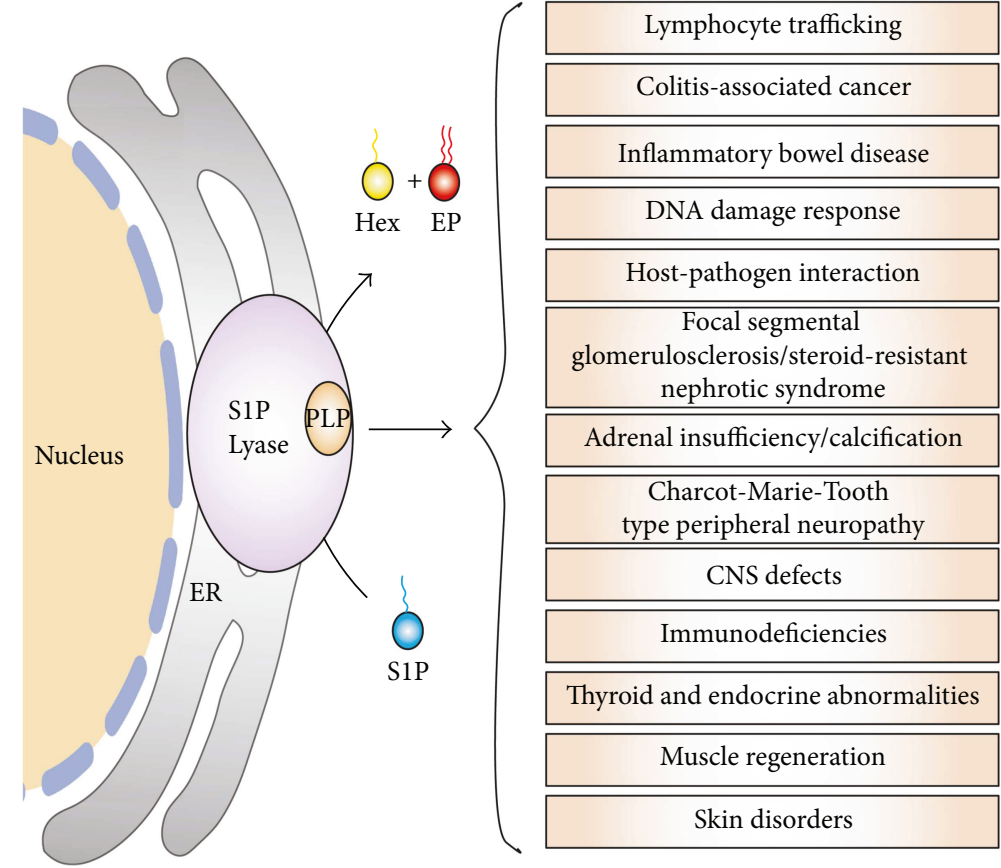

(a)

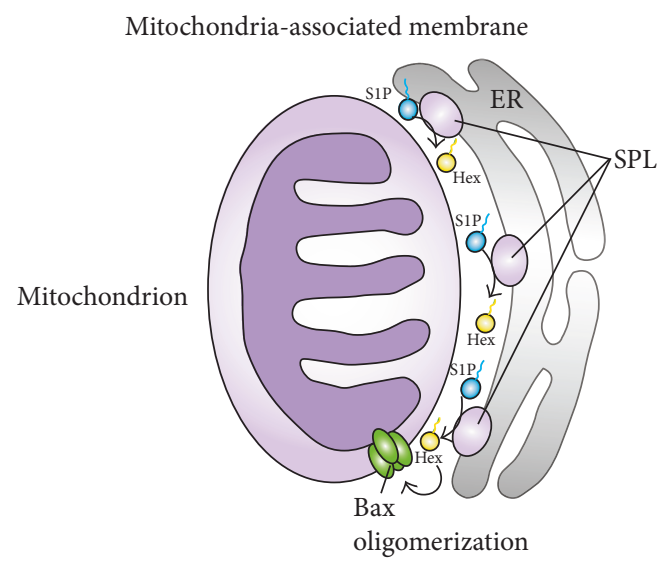

(b)

Figure 1: (a) SPL and its related diseases. (b) Besides endoplasmic reticulum, SPL is also localized in mitochondria-associated membrane (MAM). SPL present in MAM generates hexadecenal (Hex), which induces Bax activation.

central and peripheral nervous system functions, inflammation, and carcinogenesis (Figure 1(a)) [9-21]. The majority of these effects can be attributed to the ability of SPL to regulate S1P receptor signaling. However, it should be noted that some of SPL's activities may be associated with the regulation of intracellular S1P effects and with effects mediated by its products or upstream sphingolipid intermediates [22-25].

The immunological role of S1P/S1PR1, and specifically its regulation of $\mathrm{T}$ cell egress from the thymus and lymphoid organs, is the most well-characterized function of the S1P signaling pathway. S1PR1 expression on the surface of $\mathrm{T}$ lymphocytes and the presence of an S1P chemical gradient between lymphoid tissues and blood/lymph are the two essential conditions needed for lymphocyte egress from the thymus and secondary lymphoid organs [26]. Either antagonism of S1PR1 by pharmacological agents such as FTY720 or disruption of S1P gradients through SPL inhibition by 2-acetyl-4-(tetrahydroxybutyl) imidazole (THI) or its analogs block the exit of lymphocytes from the thymus and lymphoid organs, leading to lymphopenia (a reduction in absolute lymphocyte counts in the blood) [14, 27]. By preventing $\mathrm{T}$ lymphocytes with self-reactive receptors from entering target tissues, pharmacological agents targeting the S1P/S1PR1 axis have shown efficacy in the treatment of autoimmune diseases including multiple sclerosis, rheumatoid arthritis, and inflammatory bowel disease (IBD) $[28,29]$. Although regulating $\mathrm{T}$ cell egress is its most well-studied immunological function, S1P also regulates other immune functions including migration of B cells, neutrophils, macrophages, natural killer (NK) cells, and hematopoietic stem cells (HSCs). Furthermore, the development and differentiation of leukocytes, mast cell degranulation, and antigen presenting cell functions are also influenced by S1P signaling [30, 31].

A link between inflammation and carcinogenesis has been appreciated for over a century [32]. It has been suggested that inflammation can promote carcinogenesis by augmenting metastasis, angiogenesis, metabolic changes favorable to cancer cells, and resistance to chemotherapy $[33,34]$. S1P signaling has been shown to activate two critical transcription factors, nuclear factor kappa $\mathrm{B}(\mathrm{NF} \kappa \mathrm{B})$ and signal transducer and activator of transcription 3 (STAT3). Both of these transcription factors regulate the transcription of large sets of genes involved in inflammation, proliferation, and carcinogenesis. Recent studies have demonstrated the role of S1P and SPL in mediating the transition from inflammation to carcinogenesis via these signaling hubs, specifically in the context of IBD and the associated phenomenon of colitis-associated cancer (CAC).

The current review is focused on the role of SPL in regulating thymic egress and procarcinogenic inflammatory signaling. We also summarize important new insights regarding S1P metabolism, SPL structure, and recently recognized functions of SPL in the context of embryonic development and the pathophysiology of human disease. We also discuss progress in the development of SPL inhibitors and their potential therapeutic applications.

\section{Biosynthesis and Catabolism of S1P}

S1P is synthesized by two highly homologous sphingosine kinases, known as sphingosine kinase 1 (SphK1) and sphingosine kinase 2 (SphK2) [35]. SphK1 is mainly localized in the cytosol, whereas SphK2 is localized in the nucleus 

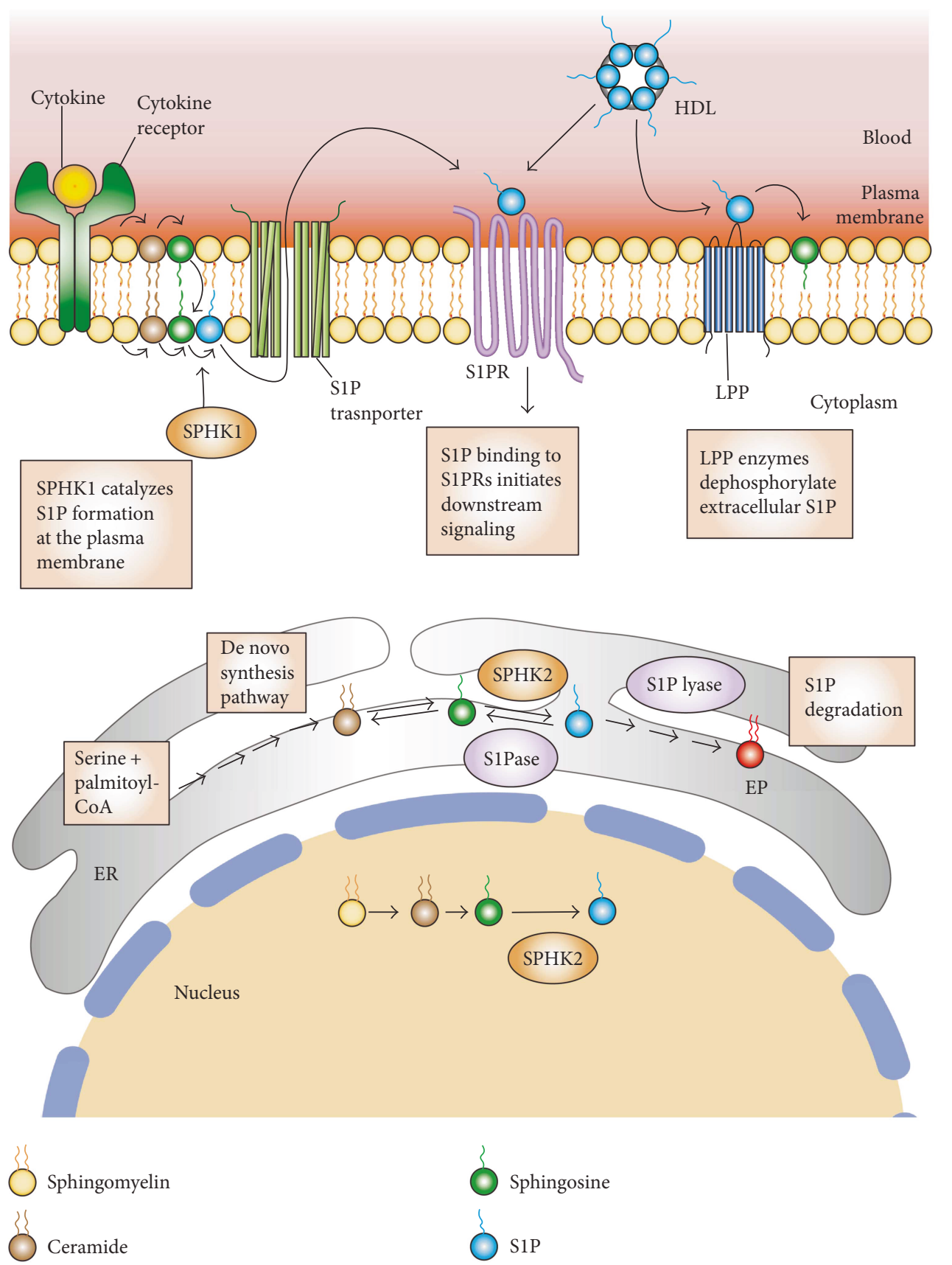

Figure 2: S1P metabolism, synthesis, and inside-out signaling. Sphingosine-1-phosphate (S1P) is irreversibly degraded by S1P lyase into ethanolamine phosphate (EP) and trans-2-hexadecenal (Hex) and dephosphorylated to sphingosine by S1P phosphatase (S1Pase) in the endoplasmic reticulum (ER) and by nonspecific lipid phosphate phosphatases (LPP) in the plasma membrane. Extracellular sphingosine formed from S1P by LPPs can be taken up by cells and be converted back to S1P or other sphingolipids. In the blood, S1P is produced primarily by erythrocytes and transported bound to albumin and high-density lipoprotein (HDL). Extracellular S1P can activate S1P receptors (S1PRs). S1P is synthesized by sphingosine kinase 1 (SPHK1) in the plasma membrane by phosphorylating sphingosine and by SPHK2 at the ER and nucleus. S1P is produced in the plasma membrane in response to stimuli and is released by specific transporters to the extracellular space where it can bind to S1PRs and initiate downstream signaling pathways (inside-out signaling).

and mitochondria-associated outer membrane (Figure 2) $[25,36]$. S1P levels in the tissues, blood, and lymph are tightly regulated by six catabolic enzymes. Two S1Pspecific phosphatases, namely, S1P phosphatase-1 (SGPP1) and S1P phosphatase-2 (SGPP2), are located in the endoplasmic reticulum (ER) dephosphorylate $\mathrm{S} 1 \mathrm{P}$, regenerating sphingosine [37, 38]. Three plasma membrane-bound lipid phosphate phosphatases (LPP1-3) [5, 39-41] dephosphorylate a broad range of lipid phosphate substrates including S1P and ceramide-1-phosphate [27, 42]. Although the LPPs do degrade a variety of lipid phosphate substrates when assayed in vitro, there does appear to be some 
specificity. LPP3 is more active against S1P or FTY720P than LPP1 and LPP2 [43-45]. Extracellular sphingosine formed from S1P by LPPs can be taken up by cells and be converted back to S1P or other sphingolipids. In contrast to the actions of the lipid phosphatases, which regenerate the substrate for SphK enzymes and thereby allow for reformation of S1P, the intracellular enzyme SPL catalyzes the irreversible degradation of S1P into ethanolamine phosphate and trans-2-hexadecenal (Figure 2) [9]. This distinction makes SPL a key factor in regulating $\mathrm{S} 1 \mathrm{P}$ levels and chemotactic gradients, as described below.

\section{Structure and Functions of SPL}

3.1. SPL Structure and Mechanism of Action. SPL is a pyridoxal 5 '-phosphate- (PLP-) dependent enzyme that belongs to the family of carbon-carbon lyase family of aldehydelyases (EC 4.1.2.27). SPL cleaves S1P between carbon atoms 2 and 3, generating trans-2-hexadecenal and ethanolamine phosphate [46]. SPL demonstrates a high degree of stereospecificity towards its substrates, cleaving naturally occurring D-erythro (2D, 3D configuration) long-chain base phosphates. However, it is not specific for chain length, degree of unsaturation, and branching of hydrocarbon chain. It can cleave S1P, dihydro-S1P, phyto-S1P, methyl-S1P, and likely also the phosphorylated form of sphingadienes, unique sphingoid bases containing two double bonds $[8,47,48]$.

The SPL gene was first identified in budding yeast and named DPL1 (for dihydrosphingosine phosphate lyase, one of the natural substrates formed in yeast cells) [49]. Subsequently, homologs have been reported in many species including mammals, insects, protozoa, bacteria, and plants $[7,8,22,50-56]$. SGPL1, the human SPL gene, encodes a protein consisting of 568 amino acids with a predicted molecular weight of $63.5 \mathrm{kDa}$ [8]. Human SPL shows $84 \%$ identity and $91 \%$ similarity in amino acid sequence with its mouse homolog [57]. Consistent with the high level of sequence homology among eukaryotic SPL proteins, all homologs that have been tested are capable of complementing a yeast $d p l 1$ mutant strain in synthetic lethal screens and other functional assays.

Human SPL predominantly resides in the ER [58]. It has also been reported to exist in the mitochondria-associated membrane [25]. The N-terminus of the SPL protein is situated in the ER lumen, whereas its active site is exposed to the cytosol [59]. Mammalian and budding yeast SPL are single-pass transmembrane ER resident proteins. Bourquin and colleagues resolved the structure of a bacterial SPL (StSPL) from Symbiobacterium thermophilum as well as a truncated form of DPL1 [56]. Based on the crystal structure of DPL1 and StSPL, they proposed a mechanism of S1P cleavage by the SPL-PLP holoenzyme that involves the transient formation of a PLP-S1P adduct $[46,56]$. SPL enzymes, DPL1 (yeast), and StSPL (bacteria) function as a dimer [56]. In contrast to DPL1, StSPL lacks a transmembrane domain, and recombinant StSPL is active in vitro and in vivo as StSPL has been shown to cleave S1P present in cell culture medium and blood [60].
3.2. Tissue Distribution of SPL. Mammalian SPL is expressed in many tissues, as shown by analysis of gene and protein expression surveys. To further investigate the tissue distribution of SPL, SGPL1 reporter mice expressing LacZ under the control of the SGPL1 promoter were generated [61]. $\beta$ Galactosidase staining of whole organs and tissue sections revealed that SPL is highly expressed in the thymus, liver, stomach, jejunum, ileum, cecum, colon, brain, spinal cord, trigeminal nerve ganglion, kidney, bladder, skin, preputial gland, Harderian gland, pituitary gland, ribcage, brown adipose tissue, adrenal cortex, ovary, and testis [61]. In contrast, SPL is faintly expressed in the tongue, esophagus, and duodenum, pancreas, heart atrium and ventricle, spleen, quadriceps muscle, sciatic nerve, lung, trachea, aorta, diaphragm, mammary gland, lacrimal gland, mesenteric adipose tissue, penis, prostate, and vesicular gland [61]. SPL is also expressed in the immune cells and secondary lymphoid organs. In the spleen, SPL is expressed in both splenocytes and stromal cell fractions. However, in the thymus, SPL is strongly expressed in thymic stromal cells, whereas its expression in thymocytes is barely detectible. SPL is also expressed in circulating leukocytes, including B and T lymphocytes, monocytes, and granulocytes [61]. Erythrocytes and platelets lack SPL expression $[62,63]$.

SPL expression begins during early developmental stages including in the neural tube and developing brain, Rathke's pouch, first brachial arch, third brachial arch, optic stalk, midgut loops, and lung buds. Strong SPL expression was observed at embryonic day 18 in the nasal epithelium, intestinal epithelium, skin, cartilage, thymus, and pituitary gland [64]. The ubiquitous expression pattern of SPL suggests it plays an important role in the function of many types of cells and tissues. In leukocytes, the main function of SPL is likely the control of cell trafficking through S1P signaling. Similarly, SPL may be important for cell migrations that occur during development. However, SPL likely has other functions. High expression of SPL in certain tissues has been linked to their rapid cell turnover. This is exemplified by the high SPL expression observed in epithelial cells of small intestine and in the mucosal cells of the olfactory bulb, both of which exhibit a high turnover rate $[65,66]$. In other tissues, high levels of SPL activity may be required to accommodate active sphingolipid recycling such as in the brain and skin, to generate products essential for organ function, or to control calcium homeostasis or cholesterol trafficking [67].

3.3. SPL in Development and Disease. In simple metazoans such as fruit flies and nematodes, SPL plays a critical role in the development $[50,51]$ (reviewed in $[57,66]$ ). Mice that completely lack SPL activity due to targeted disruption of SGPL1 fail to thrive and do not survive beyond the weaning period, exhibiting impaired lymphocyte and neutrophil trafficking, elevated cytokines and serum lipids, increased lipid storage in the liver, and deficient adipose stores [68, 69]. SGPL1 null mice also develop myeloid cell hyperplasia and significant lesions in the heart, lung, bone, and urinary tract to variable degrees [70]. Humanized SGPL1 knock-in mice exhibit $10-20 \%$ of SPL enzyme activity compared to wildtype mice. This partial restoration of SPL activity is sufficient 
to protect humanized SPL mice from the lethal nonlymphoid lesions that develop in SGPL1 null mice [70]. However, humanized SPL mice remain lymphopenic, which suggests that lymphocyte trafficking is exquisitely sensitive to alteration in the S1P levels in the thymus and lymphoid organs [70].

There is evidence to suggest that a dynamic balance between S1P and ceramide is maintained within the cells, contributing to the determination of cell fate in response to stress. SPL has the ability to promote cell death by attenuating the cell survival and proliferation signals mediated by S1P [42]. SPL plays a role in stress responses [71]. Overexpression of SPL in several malignant and nonmalignant cells has been shown to sensitize these cells to DNA-damaging drugs $[11,12]$. Conversely, SPL-deficient cells exhibit resistance to nutrient deprivation, heat shock, chemotherapeutic drugs, and radiation [12, 72-75].

Consistent with a role for S1P in carcinogenesis, SPL expression is altered in a number of cancers. SPL expression and enzyme activity are downregulated during intestinal tumorigenesis in $\mathrm{APC}^{\mathrm{Min} /+}$ mice and in tumors from colon cancer patients [11]. While this may be an indirect result of the dedifferentiation of neoplastic tissues that normally express high SPL levels, it nonetheless influences local S1P levels and can thereby promote inflammation and carcinogenesis as described below. Downregulation of SPL expression has also been reported in prostate cancer and oral squamous cell carcinoma (OSCC) [74, 76]. In contrast, upregulation of SGPL1 mRNA has been reported in OSCC, hepatocellular carcinoma, and ovarian cancer [77-79]. The etiology of this finding and its impact on carcinogenesis remain to be clarified.

S1P serves as a muscle trophic factor that enables efficient muscle regeneration. SPL is dynamically upregulated in skeletal muscle after injury but is otherwise undetectable in resting skeletal muscle [13]. We have further shown that S1P activates quiescent satellite cells (SC) via an S1PR2/ STAT3/Rac1-dependent pathway, thereby facilitating skeletal muscle regeneration [13]. Upregulation of SPL and a decrease in S1P have also been observed in the skeletal muscle of $m d x$ mice, a model for muscular dystrophy. Administration of THI to mice through drinking water raised skeletal muscle S1P levels, enhanced SC recruitment, and improved $m d x$ skeletal muscle regeneration [13].

SPL has been implicated in various lung diseases such as acute lung injury, pulmonary fibrosis, pulmonary arterial hypertension, and cystic fibrosis [80-82]. Suppression of SPL activity by THI reduces lipopolysaccharide-induced lung injury and inflammation in mice [82]. SPL deficiency in the hematopoietic compartment leads to monocytosis, impaired chemokine-induced monocyte trafficking, and monocyte differentiation into macrophages with a proinflammatory phenotype. Collectively, these effects result in an attenuated atherogenic response in low-density lipoprotein (LDL) receptor knockout (KO) mice with hematopoietic SPL deficiency [19]. S1P has been shown to promote cardiomyocyte survival and contribute to ischemic preconditioning. SPL activity is increased in heart tissue after ischemia, and mice heterozygous for an SGPL1 null allele exhibit reduced infarct size and increased functional recovery compared to wild-type mice [15]. Billich et al. generated inducible SGPL1 KO mice exhibiting partial reduction of SPL activity. These mice are protected from neurological injury in the EAE model of multiple sclerosis, and the finding was associated with reduced $\mathrm{T}$ cell immigration into the central nervous system (CNS) [18].

SPL also plays an important function in host-pathogen interactions. Legionella pneumophila is an intracellular pathogen that can cause severe pneumonia in humans. In L. pneumophila, SPL (LpSpl) has been identified as an effector protein that is translocated into the host cell by the pathogen's Dot/Icm type IV secretion system. LpSpl targets the host sphingolipid metabolic pathway and reduces sphingosine levels leading to inhibition of macrophage autophagy [53, 83]. Recently, two secretory isoforms of SPL were identified and characterized in Burkholderia pseudomallei and Burkholderia thailandensis, facultative intracellular bacteria. SPL-deficient mutants of Burkholderia sp. displayed impaired intracellular replication in murine macrophages $[84,85]$. The findings suggest that the bacterium secretes SPL to remove host-generated intracellular S1P, which somehow facilitates its survival and replication.

Recently, inherited recessive mutations in SGPL1 have been linked to human diseases representing unique inborn errors of metabolism in four separate studies [20, 21, 86, 87]. The disease-causing mutations are truncating, nonsense mutations or missense mutations in highly conserved coding regions associated with cofactor binding and catalytic activity. Lovric et al. identified nine different recessive mutations in patients affected with steroid-resistant nephrotic syndrome (SRNS) [86]. All the detected mutations led to reduced or undetectable levels of SPL protein and undetectable enzyme activity in the affected patients' fibroblasts. SGPL1 mutations were associated with SRNS and facultative ichthyosis, adrenal insufficiency, immunodeficiency, and neurological defects [86]. Janecke et al. identified two homozygous truncating mutations in association with congenital nephrotic syndrome, adrenal calcifications, and hypogonadism [20]. Prasad et al. identified four homozygous SGPL1 mutations that were associated with SRNS, ichthyosis, primary hypothyroidism, neurological symptoms, and cryptorchidism [21]. In a separate study, mutations in SGPL1 were found in association with two patients presenting with an atypical form of axonal peripheral neuropathy [87]. SGPL1 knockout mice have been shown to recapitulate the main characteristics of the human disease with abnormal adrenal and renal morphology [21]. Vascular alterations were present in a patient's renal biopsy, in line with changes seen in SGPL1 knockout mice that are compatible with a developmental defect in vascular maturation, a classical function of S1P [20]. Podocytes are known to exhibit S1PRs, and as components of the glomerular filtration barrier, they could exhibit increased sensitivity to circulating S1P levels, especially under conditions of reduced SPL activity. Two recent reports from the van Echten-Deckert laboratory also point to complex mechanisms by which SGPL1 disruption influences CNS biology, one involving a product of the reaction (ethanolamine phosphate) affecting autophagy in neurons and another showing SPL loss which 
causes S1P accumulation, leading to calcium changes in neurons thereby inducing ubiquitin proteasome-mediated effects. These studies show the many ways in which disruption of the final enzyme of sphingolipid metabolism can cause phenotypes $[88,89]$.

3.4. Functions of SPL Products. Although most of the functions and phenotypes associated with SPL and its deficiency have been attributed to its regulation of S1P levels, there is evidence that the products of the reaction catalyzed by SPL may have additional biological functions [24]. For example, these products have been shown to promote cell proliferation through an S1P-independent pathway in F9 embryonal carcinoma cells [90]. Trans-2-hexadecenal induces cytoskeletal reorganization and modulates histone acetylation pattern thereby inducing the transcription of inflammatory cytokines [23, 24]. Furthermore, trans-2-hexadecenal reacts readily with deoxyguanosine and DNA to produce the diastereomeric cyclic $1, \mathrm{~N}(2)$-deoxyguanosine adducts 3-(2deoxy- $\beta$-d-erythro-pentofuranosyl)-5,6,7,8-tetrahydro-8Rhydroxy-6R-tridecylpyrimido[1,2-a]purine-10 $(3 \mathrm{H})$ one and 3-(2-deoxy- $\beta$-d-erythro-pentofuranosyl)-5,6,7,8-tetrahydro$8 \mathrm{~S}$-hydroxy-6S-tridecylpyrimido[1,2-a]purine-10(3H)one [91]. Trans-2-hexadecenal forms Michael adduct with glutathione and with seven amino acids [92]. It also binds directly to Bax, leading to a conformational change in Bax, resulting in its activation, which induces apoptosis (Figure 1(b)) [24, 25]. It is not surprising that the longchain aldehyde product interacts with many targets, as aldehydes are known to interact with proteins, nucleic acids, and carbohydrates. Ethanolamine derived from the SPL reaction was shown to be required for stationary phase differentiation and virulence in Leishmania [22]. Unlike other organisms that depend on sphingolipid biosynthesis and degradation for signaling and membrane structure/function, this organism depends on sphingolipid degradation primarily as its major route for ethanolamine synthesis. This raises the possibility that inhibiting SPL could be a useful strategy for the treatment of this parasitic disease.

\section{Regulation of Lymphocyte Trafficking by S1P/S1PR1}

S1P signaling has emerged as a central mediator of the trafficking of hematopoietic cells including lymphocytes, NK cells, neutrophils, dendritic cells (DCs), macrophages, hematopoietic progenitors, and mast cells [93-102]. Most evidence points to the role of S1P signaling in the control of hematopoietic cell egress from bone marrow, tissues, and lymphoid organs. However, there are instances where S1P signaling also regulates the entry of cells into the parenchyma, such as $\mathrm{T}$ cell entry into inflamed tissues [103]. S1PRs are expressed in all recognized immune cell types, although each cell type expresses only a subset of S1PRs. S1PR1 is expressed in most immune cells, whereas S1PR5 is expressed primarily by DCs and NK cells. S1PRs appear to have unique cellspecific functions. S1PR1 is most widely recognized for its crucial role in $\mathrm{T}$ and $\mathrm{B}$ lymphocyte trafficking, including the egress of mature $\mathrm{T}$ cells from the thymus and peripheral lymphoid organs [26].

4.1. S1PR1 Signaling and Recycling in T Cell Egress. Early thymic progenitor cells originating in the bone marrow enter the thymus at the corticomedullary junction and move into the cortex where they differentiate into $\mathrm{T}$ cell receptor(TCR-) expressing $\mathrm{CD} 4^{+} \mathrm{CD} 8^{+}$double-positive (DP) thymocytes (Figure 3 ). DP thymocytes undergo positive selection, a process in which thymocytes with inefficient $\mathrm{T}$ cell receptors are culled. Surviving thymocytes differentiate into semimature CD4 or CD8 single-positive (SP) cells that express CCR7. This population migrates to the medulla to undergo negative selection, a process in which thymocytes with selfrecognizing $\mathrm{T}$ cell receptors are culled $[104,105]$. The small number of semimature cells that have survived both positive and negative selections upregulates the transcription factor Krüppel-like factor 2 (KLF2), which promotes the transcription of S1pr1 as well as other genes involved in homing, including CD62L [106, 107]. Klf2 itself is also transcriptionally regulated by several factors including Foxo1 and PI3K/ Akt [108, 109]. Activation of cell surface-localized S1PR1 by S1P enables the mature $\mathrm{T}$ cells to egress the thymus and enter the circulation via blood vessels located at the corticomedullary junction (CMJ) $[3,110]$. Binding of S1P to S1PR1 on mature $\mathrm{T}$ cells activates RhoA GTPase and induces polarization of the actin cytoskeleton, as well as integrin clustering and activation. Mst1 and Mst2 kinases regulate the activation of RhoA and the migratory responses of SP thymocytes [111, 112]. Mst1/Mst2 kinases appear to act downstream of S1PR1 and upstream of RhoA, as mice lacking both kinases exhibit a block in thymic egress and show defects in RhoA activation and polarization of the actin cytoskeleton [112].

S1pr1 mRNA expression is upregulated as thymocytes mature. A 50-fold increase is observed between DP and SP stages, while a 30 -fold increase has been noted between immature and mature SP stages [26]. A defect in T cell egress and a concomitant depletion of mature $\mathrm{T}$ cells in the periphery has been noted in mice lacking S1pr1 or Klf2 in $\mathrm{T}$ lymphocytes [26, 106, 113]. Conversely, in a gain-offunction approach, either premature expression of S1PR1 in immature thymocytes or S1PR1 expression in thymocytes lacking Klf2 was sufficient to drive their egress from the thymus, confirming that S1pr1 is the main target of KLF2 needed for thymic egress [98].

Membrane expression of S1PR1 is negatively regulated by CD69, a lymphocyte activation marker. CD69 directly interacts with S1PR1 in a conformation that mimics some aspects of the ligand-bound state and promotes internalization and degradation $[114,115]$. Mature SP cells express intermediate levels of CD69, but S1P signaling causes rapid downmodulation of surface CD69 [116]. Surface expression of S1PR1 on T cells is exquisitely sensitive to exposure to S1P. Nanomolar concentrations of S1P are sufficient to induce S1PR1 internalization. In response to persistent S1P signal, S1PR1 present on the $\mathrm{T}$ cell plasma membrane is phosphorylated by GPCR kinase-2 (GRK2) [117, 118]. Grk2-deficient T cells are resistant to S1P-mediated S1PR1 
Thymus
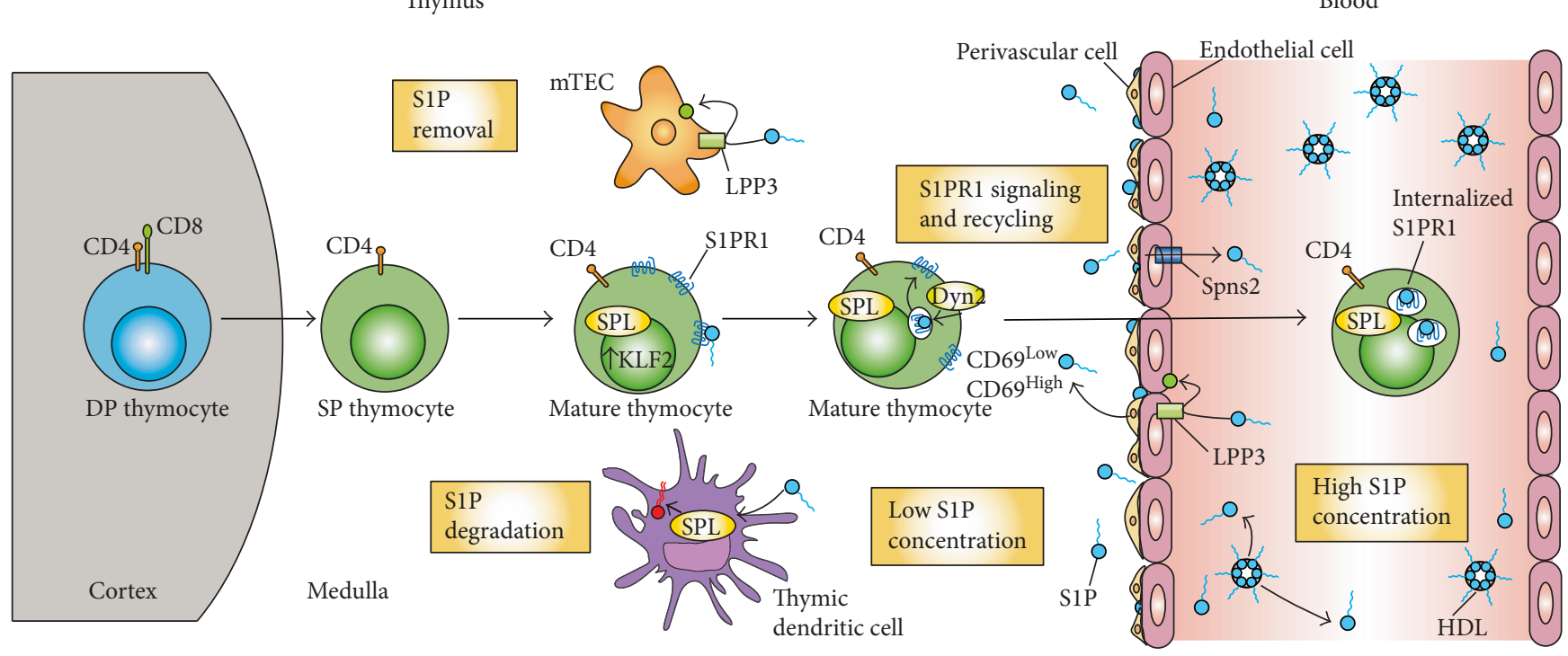

Blood

FIGURE 3: Egress of mature thymocytes from the thymus. Double-positive (DP) CD4 ${ }^{+}$and $\mathrm{CD} 8^{+}$thymocytes migrate from the thymic cortex to the medulla, where they differentiate into $\mathrm{CD}^{+}$and $\mathrm{CD}^{+}$single-positive (SP) thymocytes. As these cells mature, they express S1P lyase (SPL) and the transcriptional factor KLF2. The latter leads to increase transcription of its target gene, s1pr1, resulting in a high expression of surface S1PR1. Perivascular and endothelial cells in the corticomedullary region secrete S1P. Thymic dendritic and medullary thymic epithelial cells remove extracellular S1P by irreversible degradation or dephosphorylation to sphingosine, respectively. The resulting small S1P gradient interacts with the S1PR1 on the mature thymocytes. Prior to exit, S1PR1 receptors undergo multiple cycles of internalization to finally exit into the blood where there is a high S1P concentration. The high S1P concentration leads to S1PR1 permanent internalization and degradation.

downmodulation even at micromolar concentrations [118]. S1PR1 phosphorylation recruits $\beta$-arrestins that dissociate the receptor from heterotrimeric $G$ proteins, leading to receptor desensitization by terminating the signaling cascade. Recruitment of arrestins also causes S1PR1 internalization via clathrin-mediated endocytosis. Internalized S1PR1 can be degraded or recycled back to the cell surface. Binding of S1P to S1PR1 triggers activation of $G$ proteins, thereby leading to effector function, but it also leads to receptor endocytosis $[119,120]$. Receptor internalization through endocytosis and subsequent recycling is thought to be essential for restoring the responsiveness of S1PR1 [121, 122].

It has been proposed that the S1PR1 receptor desensitization (termination of S1P signaling) occurs via a GRK2mediated pathway, whereas resensitization (recycling of S1PR1) occurs via dynamin 2-dependent endocytosis [123]. The in vivo response of S1PR1 appears to vary in different contexts, namely, in the presence of different concentrations of S1P. In an S1P-high environment such as the blood, receptor internalization is followed by its degradation, thereby terminating S1PR1 signaling and resulting in permanent desensitization. In contrast, in an S1P-low environment such as exit sites in the thymus, S1PR1 internalization does not trigger its degradation but instead results in continuous signaling via receptor recycling, ultimately facilitating $\mathrm{T}$ cell egress [123]. Both of these pathways are reciprocally regulated by GRK2 and a GTPase, dynamin 2. Although dynamin 2 is not required for the termination of S1PR1 signaling, dynamin-dependent endocytosis is required for S1PR1 resensitization in T lymphocytes. Dynamin 2 may help in maintaining sustained S1P-S1PR1 signaling in low
S1P environments including the thymic CMJ, where T cells egress into the blood [123]. Mice lacking $\mathrm{T}$ cell dynamin 2 accumulate mature $\mathrm{T}$ cells in the thymus and exhibit profound lymphopenia [123]. Gatfield et al. [124] have shown that SPL is important for S1PR1 recycling in human umbilical vein endothelial cells by removing S1P from the receptor. Thus, SPL intrinsic to $\mathrm{T}$ cells may also be required for S1PR1 recycling and sustained S1P/S1PR1 signaling in mature T cells.

4.2. S1P Export and Transport. Erythrocytes and platelets are major contributors to blood S1P, as both of these cell types are exposed to circulating sphingosine, contain SphKs activity, and lack most of the S1P-degrading enzymes [30, 62]. Circulating S1P is cleared rapidly; the half-life of albuminbound S1P in blood is $\sim 17 \mathrm{~min}$. S1P in the lymph is produced by radiation-resistant cells of nonhematopoietic origin, presumably lymphatic endothelial cells [125-127]. Mice lacking both isoforms of SphK in hematopoietic cells have undetectable S1P levels in plasma and lymph and exhibit a block in $\mathrm{T}$ cell egress from the thymus and lymph nodes [127]. Restoration of S1P content in the plasma of Sphk knockout mice (by infusion of erythrocytes from wild typemice) rescued their lymphopenic phenotype by permitting thymic egress. These experiments confirm the requirement of plasma S1P for thymic egress [127].

After synthesis, S1P can be exported from the cell (Figure 2). S1P is an amphipathic molecule that requires facilitated export. The ATP-binding cassette (ABC) family of transporters including ABCA1, ABCC1, and ABCG2 has been shown to export S1P [128-130]. Erythrocytes and 
endothelial cells export S1P constitutively in a stimulusindependent manner. In contrast, mast cells and platelets secrete S1P in a stimulus-dependent manner and require the activity of ABCC1- and ABCA-like transporters, respectively. Another important regulator of extracellular S1P is the transporter Spns2, which has been shown to control S1P levels in the plasma and lymph by regulating S1P release from endothelial and lymphoendothelial cells [54, 131-133]. Global Spns2 KO mice phenocopy S1pr1 KO mice and exhibit a block in thymic egress and lymphopenia. Furthermore, Spns2-mediated export of S1P from endothelial cells is required for producing the S1P gradients required for the egress of $\mathrm{B}$ and $\mathrm{T}$ lymphocytes from the bone marrow and thymus, respectively $[134,135]$. Once in the extracellular space, S1P can bind and activate the S1PRs present on neighboring cells (paracrine action) or on the same cell (autocrine action). The latter mechanism is also known as "inside-out signaling" [35].

In the blood, most of the plasma S1P is transported bound to high-density lipoprotein (HDL) (50-60\%). The remaining part binds to albumin (30-40\%), LDLs ( 8\%), and very low-density lipoproteins (2-3\%). Apo M (ApoM), a lipocalin protein, is the carrier of S1P in HDL (Figure 2). ApoM acts as an S1P chaperone and may additionally protect S1P from degradation and facilitate its presentation to receptors [125, 136]. Albumin-bound S1P and free S1P are susceptible to degradation, whereas HDL-bound S1P is more stable [125]. Levels of free S1P may be at least 40 times lower than the total S1P concentrations [125]. Many of the beneficial effects of HDL have been ascribed to its ApoM-S1P component [137].

4.3. Role of the S1P Gradient in Thymic Egress. Any chemotactic signal requires the presence of a chemical gradient in the local environment and a cellular receptor that senses that chemical gradient and stimulates reorganization of the cellular cytoskeleton, enabling movement toward or away from the gradient. A requirement for SPL and an S1P gradient in regulating $\mathrm{T}$ cell egress was first revealed by Schwab and colleagues [14]. They showed that pharmacological inhibition or genetic disruption of SPL causes $\sim 1000$-fold elevation in thymic S1P levels and disturbs the S1P gradient between parenchyma and the exit site, leading to a block in $\mathrm{T}$ cell egress $[14,70]$. Immunosuppressant drugs such as FTY720 and THI work precisely by disrupting S1P signaling [14, 28, 138, 139]. FTY720 is a prodrug that is phosphorylated in vivo by SphK2 to become the active drug. The phosphorylated form, which is an analog of S1P, behaves as a super ligand; it tightly engages and sequesters S1PR1, 3, 4, and 5, thus inhibiting any downstream signaling. THI, on the other hand, inhibits SPL and prevents S1P degradation, thereby leading to increases in intracellular and extracellular S1P levels (Figure 2) [14]. Both drugs prevent the exit of $\mathrm{T}$ and $\mathrm{B}$ cells from secondary lymphoid organs and the thymus [28, 138, 139].

The S1P signal for T cell egress is produced on the front end via the synthesis and export of S1P by neural crestderived pericytes surrounding thymic vascular endothelial cells [98]. We have recently shown that thymic dendritic cells
(DCs), located at the CMJ, are responsible for maintaining the gradient at the back end, that is, by continuously degrading S1P in the thymic parenchyma, as described in detail below [140]. Endothelial cells also contribute to the S1P gradient by transporting S1P into the extracellular space via the Spns2 (Figure 2) [134]. Interestingly, endothelial cells may regulate the S1P gradient by removing extracellular S1P via dephosphorization through LPP3 [45]. Medullary thymic epithelial cells (TECs) described below also contribute to the low thymic S1P levels by transiently removing S1P through LPP3 (Figure 3).

4.4. Dendritic Cell SPL Generates the Chemotactic Gradient Essential for Thymic Egress. In the thymic tissue, SPL is predominantly expressed in stromal cells called TECs $[61,141]$. To ascertain the role that SPL in the TEC compartment might play in regulating $\mathrm{T}$ cell egress, we used cre/lox technology to generate mice in which SGPL1 is disrupted only in TECs. Surprisingly, we found that transgenic mice lacking TEC SPL exhibit normal T cell development, normal thymic S1P levels, appropriate levels of S1PR1 expression on mature T cells, and normal T cell egress [140]. Thus, despite the high SPL content in TECs, SPL activity intrinsic to TECs does not contribute appreciably to $\mathrm{T}$ cell egress.

Besides TECs, the thymus is largely comprised of endothelial and hematopoietic cells. To determine if SPL expression in endothelial and/or hematopoietic cell compartments is required for thymic egress, transgenic mice lacking SPL in these compartments (SPL ${ }^{\mathrm{Mx1KO}}$ mice) were generated. When these mice are induced with polyinosinic:polycytidylic acid, SGPL1 is disrupted in their spleen, blood, and liver. Deletion of SPL in hematopoietic and endothelial compartments using this strategy resulted in a twofold increase in CD4SP and CD8SP, as well as a fivefold increase in mature CD4SP and mature CD8SP T cells in the thymus. A concomitant depletion of $\mathrm{CD} 4^{+}$and $\mathrm{CD} 8^{+} \mathrm{T}$ cells was noted in the blood, lymph, spleen, and mesenteric lymph nodes. SPL deletion in hematopoietic and endothelial cells led to a marked increase in thymic S1P levels, accompanied by a decrease in the surface expression of S1PR1 in mature CD4SP cells [140]. These findings indicated that SPL is required in hematopoietic cells, endothelial cells, or both to establish the S1P gradient and to facilitate egress of mature $\mathrm{T}$ cells. Colocalization studies revealed that SPL is expressed in endothelial cells. We disrupted SGPL1 in vascular and lymphatic endothelial cells through targeted recombination, and the resulting mice exhibited normal thymic egress and normal numbers of lymphocytes in the blood and secondary lymphoid organs. Furthermore, we did not observe significant differences in thymic S1P levels or surface expression of S1PR1 on mature CD4SP T cells between mice lacking endothelial SPL and control mice [140].

To confirm that SPL is required in the hematopoietic cell compartment to support $\mathrm{T}$ cell egress, we performed bone marrow (BM) reconstitution experiments. When irradiated SPL $^{\text {Mx1KO }}$ mice were transplanted with the BM from wildtype mice, their thymic egress block was relieved, and they were no longer lymphopenic. Conversely, BM transplantation from $\mathrm{SPL}^{\mathrm{Mx} 1 \mathrm{KO}}$ mice to wild-type mice recapitulated 
the thymic egress defect. These data demonstrated that SPL expression in a cell type of hematopoietic origin must be required for egress [140].

To determine if SPL activity intrinsic to $\mathrm{T}$ cells enables their egress, we disrupted SGPL1 in thymocytes at the DP stage. SGPL1 disruption in T cells resulted in modest accumulation of $\mathrm{T}$ cells in the thymus and an increase in thymic S1P levels but did not phenocopy the severe thymic egress defect and peripheral lymphopenia observed in SPL ${ }^{M x 1 K O}$ mice [140]. This suggested that $\mathrm{T}$ cell SPL contributed to their egress but was not the primary compartment in which SPL generates the S1P gradient. Besides thymocytes, three other hematopoietic cell types are present in the thymus, namely, B cells, macrophages, and DCs. SPL deletion in macrophages and B cells resulted in no effect on thymic S1P levels, S1PR1 expression, or T cell egress, suggesting that SPL in these cells is not required for the maintenance of the S1P gradient.

DCs are found in the thymic medulla and at the CMJ $[98,142]$. We observed SPL expression in thymic DCs. To investigate whether DC SPL was required for egress, $S L^{\text {DCKO }}$ mice lacking SPL specifically in DCs were generated. SPL ${ }^{\mathrm{DCKO}}$ mice exhibited prominent retention of SP cells and mature $\mathrm{T}$ cells in the thymus, were lymphopenic, and exhibited markedly high thymic S1P levels. Further, their mature $\mathrm{T}$ cells showed a decrease in surface expression of S1PR1 [140]. In a rescue experiment, mice lacking SPL in DCs were injected with wild-type DCs. These DCs with normal amounts of SPL homed to the thymus of recipient mice and restored lymphocyte egress [140]. These surprising cumulative observations show that thymic DCs, which constitute only a small percentage of thymic stromal cells, are the main metabolic gatekeepers of thymocyte egress.

To ascertain how DCs lower extracellular S1P levels to produce the gradient, we exposed bone barrow-derived DCs (BMDCs) to radiolabeled S1P in vitro. BMDCs could take up extracellular S1P in an S1PR-dependent manner that was blocked by treatment with FTY720. Collectively, these findings demonstrate that DCs play a central role in thymic egress by producing the S1P chemotactic gradient at the CMJ. Prior to this study, thymic DCs were recognized primarily for their role in negative selection and central tolerance. This is the first time thymic DCs have been shown to contribute to thymocyte egress by generating a localized S1P gradient (Figure 3).

One important question raised by our findings is whether DCs circulating throughout the body modulate their SPL expression or activity in response to environmental conditions such as infection, inflammation, and exposure to toxic stimuli. If so, can those DCs influence thymic output in response to these events in the periphery? Other S1Pmetabolizing enzymes such as LPPs and ceramide synthase 2 have also been shown to regulate thymic S1P levels and thereby influence $\mathrm{T}$ cell egress $[45,143]$. Why are so many enzymes required to maintain the S1P gradient? Are there other localized gradients that are required to sustain $\mathrm{T}$ cell egress from the thymus? Is the $\mathrm{S} 1 \mathrm{P}$ gradient dynamically regulated? New methodologies may be required to answer some of these questions. Fortunately, elegant systems including reporter mice have recently been developed to facilitate the detection of S1P signaling footprints and signaling in real time in murine tissues [144]. With this novel approach, it may be possible to assess whether S1P gradients are dynamically regulated within tissues, orchestrating the movements of many cells simultaneously.

\section{S1P Signaling and Inflammation}

S1P has been implicated as a critical factor in the signaling and biological functions of inflammatory mediators such as lipopolysaccharide (LPS), tumor necrosis factor- (TNF-) $\alpha$, and interleukin- (IL-) $1 \beta$. Through the activation of its receptor, TNF- $\alpha$ induces the recruitment of TNF receptorassociated factor 2 (TRAF2), an E3 ubiquitin ligase that activates I kappa B kinase proteins, which results in $\mathrm{NF} \kappa \mathrm{B}$ signaling through the canonical pathway. Alvarez et al. demonstrated that intracellular S1P generated by SphK1 binds to TRAF2 and acts as its cofactor [145]. This study revealed the role of intracellular S1P generation in the activation of the canonical $\mathrm{NF} \kappa \mathrm{B}$ signaling pathway. In a model of bronchial asthma, S1PR2 activation by S1P increased the expression of the chemokine (C-C motif) ligand- (CCL-) 3 through the activation of $\mathrm{NF} \kappa \mathrm{B}$ and STAT3 signaling pathways [146]. Administration of JTE013, a specific S1PR2 inhibitor, reduced both $\mathrm{NF} \kappa \mathrm{B}$ and STAT3 activations resulting in decreased CCL3 expression. STAT3 can be activated through S1PR1 signaling following S1P stimulation. We showed that when SPL is silenced in mouse embryonic fibroblasts, extracellular S1P activates STAT3 through an S1PR1-dependent pathway, resulting in decreased expression of the antioncogene CYLD, a negative regulator of $\mathrm{NF} \kappa \mathrm{B}$ [147] (Figure 4). In a mouse model of chronic colon inflammation, Nguyen et al. demonstrated that the increased STAT3 signaling in epithelial cells under inflammatory conditions was a result of S1P-dependent activation of S1PR1 [148] (Figure 4). By activating epithelial STAT3, S1P signaling promoted higher expression of proinflammatory cytokines including IL-17 and IL- 6 and decreased the expression of CCL19, CCL28, and RANTES, which are regulatory $\mathrm{T}$ cell (Treg) recruiters and, therefore, have antiinflammatory functions. Flow cytometry analysis of the inflamed colon under these conditions showed increased recruitment of cytotoxic $\mathrm{CD}^{+} \mathrm{T}$ cells and reduced recruitment of Tregs [148]. $\mathrm{CD}^{+} \mathrm{T}$ cells are thought to play a key role in promoting tissue damage to the colonic epithelium and are associated with enhanced inflammation in patients suffering from IBD [149]. Tregs suppress the activity of $\mathrm{CD}^{+} \mathrm{T}$ cells by affecting granule exocytosis, thereby rendering $\mathrm{T}$ cells unable to kill their target cells [150]. This study illustrates the role of the S1P/S1PR1/STAT3 axis in the regulation of colitis. These examples demonstrate that $\mathrm{S} 1 \mathrm{P}$ signaling contributes to the inflammatory processes mainly through the activation of two key proinflammatory transcription factors, NF $\kappa \mathrm{B}$ and STAT3.

5.1. SPL Regulation of Inflammatory Signaling. SPL is crucial for attenuating S1P signaling and its effect on inflammatory pathways. Mice with an inactive SPL gene (SGPL1) die soon 


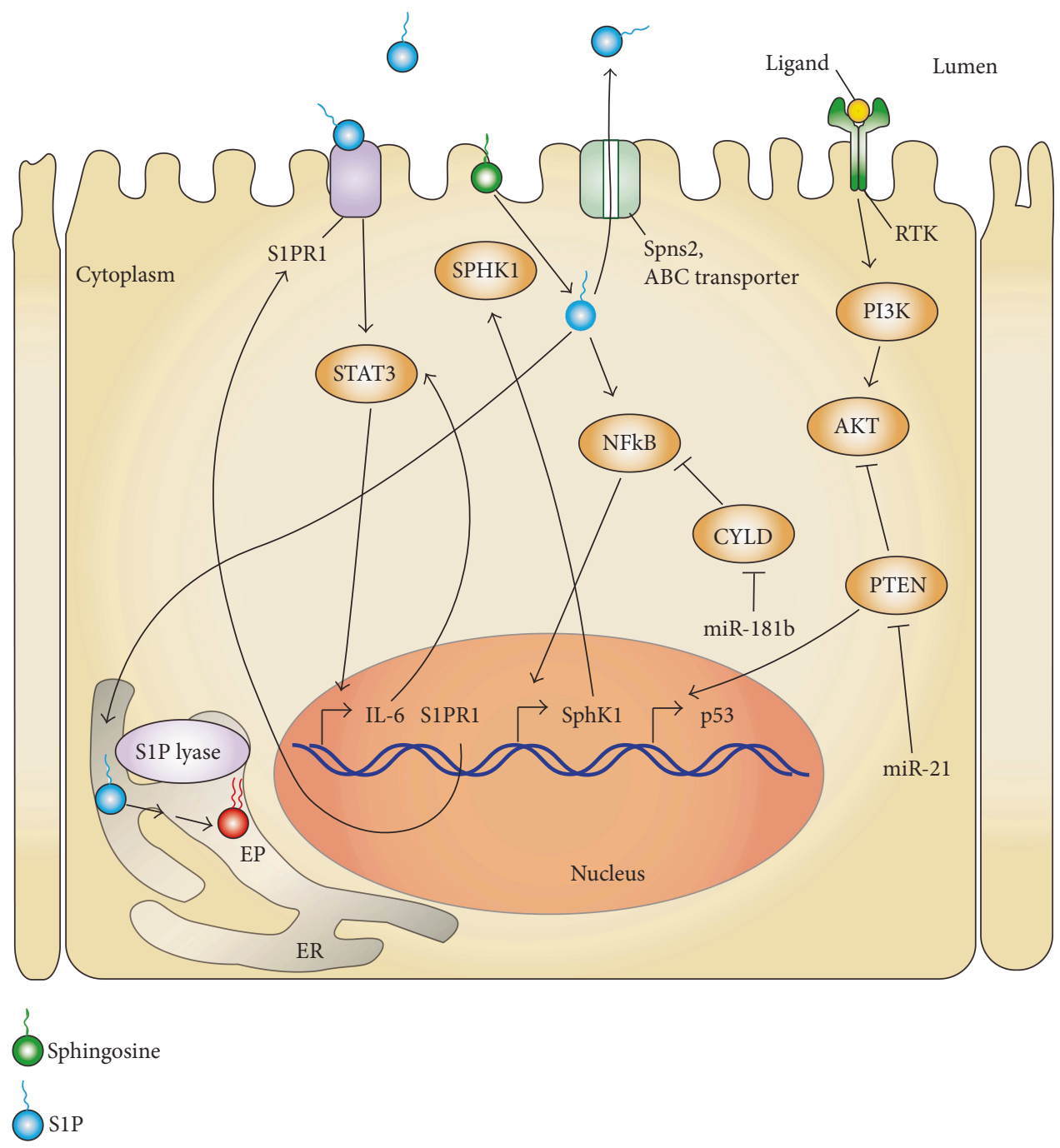

FIGURE 4: STAT3/S1P/S1PR1/SPHK1/SPL-positive feed-forward loop promoting CAC. In a chronic inflammatory milieu, STAT3 is persistently activated through two pathways. (1) SphK1 produces S1P which when kept intracellular can activate the NF $\kappa$ B pathway leading to increased expression of SPHK1. (2) When S1P is exported out of the cells via the SPNS2 transporter, the binding of S1P to its S1PR1 activates STAT3. STAT3 upregulates IL-6 and S1PR1 expression as well as miR-181b1 and miR-21. miR-181b1 decreases the expression of the tumor-suppressor CYLD which results in derepression of the NF $\kappa$ B pathway. miR-21 decreases the expression of PTEN which is an antioncogene negatively regulating the PI3K/AKT pathway and positively p53. All these pathways are interrelated in an amplification loop, permitting the persistent STAT3 activation, which is involved in cell transformation and tumor progression. Bent arrows indicate transcriptional regulation.

after weaning and have high levels of circulating and tissue S1P leading to increased levels of proinflammatory cytokines (TNF, IFN- $\gamma$, MCP-1, and IL-6) and upregulation of genes associated with acute-phase response (SAA1, SAA3) in the liver [151]. Similarly, a role for SPL has been established in cardiomyocytes in a study of mice treated with the SPL inhibitor THI [152]. The mice treated with THI have higher levels of S1P in cardiac tissue resulting in aggravated pathological cardiac remodeling after myocardial infarction. In this context, S1P activation of S1PR1 activated both NF $\kappa$ B and STAT3 pathways increasing the expression of proinflammatory cytokines TNF- $\alpha$ and IL- 6 , which could be reverted by blocking S1PR1 with the inhibitor W146. These studies highlight an anti-inflammatory role for SPL. However, a recent paper from Ebenezer et al. demonstrated a proinflammatory role for SPL [23]. In an in vitro model of LPS-induced lung inflammation, the authors demonstrated that the end products of S1P degradation by SPL (trans-2-hexadecenal and phosphoethanolamine) modulated the histone acetylation profile in a different way than that mediated by nuclear S1P produced by SphK2 [23]. Blocking SPL resulted in modified HDAC acetylation patterns and decreased secretion of proinflammatory cytokines normally induced following LPS treatment in lung endothelial cells. These results are in accordance with a paper by Zhao et al. showing that intratracheal instillation of LPS to mice increased the levels of SPL leading to decreased levels of S1P in the lung tissue which ultimately resulted in lung inflammation and injury. Inhibiting SPL with THI had a protective effect resulting in less lung inflammation and injury. The increased lung S1P 
level attenuated phosphorylation of p38-MAPK and I- $\kappa \mathrm{B}$, IL-6 secretion, and endothelial disruption normally attributed to LPS treatment [82].

These studies indicate that manipulating SPL and S1P for therapeutic purposes may represent some challenges. Both of these targets control cell survival, barrier function, epigenetic gene regulation, and inflammatory signals that may protect tissues and help maintain tissue homeostasis in the face of acute insults but may induce injury when sustained. Colitis and CAC are well-established examples of how tissue homeostasis can be lost in the face of chronic inflammatory signaling. The above findings highlight the need for further exploration of the best ways to target the S1P pathway therapeutically in a disease-dependent context.

5.2. S1P and Inflammation-Associated Cancer. Patients suffering from chronic inflammation and other autoimmune diseases are at higher risk of developing cancer than the general population [153]. The mechanism for this observation has not been fully elucidated, although many potential explanations have been proposed as elegantly reviewed by Elinav et al. [154]. It is thought that in the inflamed tissue, the production of reactive oxygen species by immune cells induces DNA damage. This insult is combined with an inflammatory milieu containing cytokines that stimulate cell survival and proliferation, primarily through the activation of the transcription factors $\mathrm{NF} \kappa \mathrm{B}$ and STAT3. The activation of these two signaling hubs by S1P can result in a change in the cell's genetic program that facilitates cell transformation and the acquisition of the cancer phenotype. S1P levels are high in chronic inflammation, and several studies have confirmed the role of S1P in the development of cancer. Lee et al. showed a positive feed-forward loop implicating STAT3 and S1P/S1PR1 signaling pathways in carcinogenesis and metastasis using mice bearing urothelial carcinoma MB49 or melanoma B16 tumor cells [155] (Figure 4). The mutual coactivation of these pathways results in the persistent activation of STAT3 which promotes carcinogenesis by upregulating the expression of IL- 6 and additional factors involved in tumor growth and metastasis. The most wellestablished examples of S1P's role in inflammationassociated cancer are in the context of CAC. Nguyen and colleagues demonstrated that when chronic inflammation is present, increased IL-6 expression leads to persistent activation of intestinal epithelial STAT3, which regulates S1P production through SphK1 [148] (Figure 4). As mentioned earlier, the resulting imbalance of cytotoxic $\mathrm{CD}^{+} \mathrm{T}$ cells versus Treg cells intensifies intestinal inflammation, thus promoting tumor progression and malignancy. Liang et al. also demonstrated that colonic inflammation could lead to CAC through a positive-feed forward loop linking persistent STAT3 activation and S1P/S1PR1 signaling [156] (Figure 4). Consistent with previous studies, they showed that SphK1 and S1PR1 expressions were increased in colitis and CAC, concomitant with an increased level of tissue S1P. They went further to show that both extracellular and intracellular S1P contributed to the development of tumorigenesis. They showed that extracellular S1P activated STAT3 through S1PR1 in a Src-dependent manner. By activating $N F \kappa B$, intracellular S1P resulted in increased expression of both IL-6 and TNF- $\alpha$, which led to further STAT3 activation and activation of SphK1. In a CAC mouse model, treatment with FTY720 to block S1PR1 activation reduced both upregulations of S1PR1 and SphK1 in the carcinomas.

\subsection{SPL Regulation of Inflammation-Associated Cancer.} Studies from our group demonstrated that SPL expression is high in the intestinal epithelium but downregulated in murine adenomas and human colorectal cancers [11, 61]. This finding, combined with a growing appreciation for the key role S1P signaling plays in colon cancer [156-159], prompted us to investigate the potential effect of gut epithelial SGPL1 disruption on the development of CAC. Toward that end, we generated an inducible intestinal epitheliumspecific SPL KO mouse (SPL ${ }^{\text {GutKO}}$ ) using Cre/lox technology $[10,147]$. SPL ${ }^{\text {GutKO }}$ mice showed an 8-fold increase in S1P levels in the small intestine and colon tissues, whereas plasma S1P levels were unchanged, demonstrating that the SPL GutKO mouse is a good model system for testing the effects of localized S1P accumulation on intestinal biology. There were no obvious changes in gut epithelial architecture, weight, litter size, or life span in SPL GutKO mice compared to "floxed" littermates lacking the Cre $\mathrm{Tg}$ used as controls. However, when SPL ${ }^{\text {GutKO }}$ mice were treated with dextran sodium sulfate (DSS) to induce a chemical colitis, they developed more severe colitis than the controls, with shortened colon length, increased disease activity, and higher levels of tissue S1P compared to DSS-treated controls. When SPL GutKO mice were treated with both the carcinogen azoxymethane and DSS to induce CAC, they exhibited an increased susceptibility to CAC compared to controls [147]. The SPL ${ }^{\text {GutKO }}$ mice exhibited reduced survival and an increased intensity of disease. They also exhibited increased levels of plasma and tissue cytokines including TNF- $\alpha$, IL-1 $\beta$, and IL-6. Importantly, these mice also were found to have higher percentages of Th17 cells and increased number of macrophages than controls. Th17 cells and STAT3-mediated inflammation correlate with autoimmune diseases like experimental autoimmune encephalitis and colitis [160, 161]. In SPL ${ }^{\text {GutKO }}$ mice, the number of tumors per mouse was higher than in controls, whereas tumor size and grade were unaffected. However, using $\mathrm{Ki} 7^{+}$staining, we observed a higher number of proliferating cells in the region surrounding the tumors in the SPL ${ }^{\text {GutKO }}$ mice. This finding prompted us to hypothesize that SPL deletion promotes an early step of the carcinogenic process, such as cell transformation. Studies by Hua Yu and colleagues that had linked S1P to STAT3 signaling in 2010 prompted us to examine this pathway in SPL ${ }^{\text {GutKO }}$ mice (Figure 4). We found that STAT3 phosphorylation was increased in SPL GutKO mice compared to control mice. In addition, the STAT3 target genes $c-M y c, M c l-1$, and S1pr1 as well as $S p h k 1$ were all increased in the nontumor colon tissue of SPL ${ }^{\text {GutKO }}$ mice compared to controls [147]. STAT3 is a transcription factor for multiple genes associated with inflammation and tumor progression, but it is also an important transcription factor for micro RNAs (miRNAs). Iliopoulos et al. demonstrated that persistent STAT3 activation leads to inflammation and cancer by increasing the 
expression of miR-181b1 and miR-21 which silence two tumor-suppressor genes Cyld and Pten, respectively [162]. PTEN is a phosphatase that acts by suppressing PI3K signaling, whereas CYLD is an ubiquitin ligase that acts by inhibiting $\mathrm{NF} \kappa \mathrm{B}$ signaling. We found that expressions of miR-181b and miR-21 were elevated, whereas their target proteins CYLD and PTEN were diminished in SPL GutKO mice colons compared to those of control mice. The increased susceptibility of SPL ${ }^{\text {GutKO }}$ mice to tumorigenesis was rescued by the administration of the STAT3 inhibitor NSC 74859. We then examined the impact of SPL silencing in mouse embryonic fibroblasts (MEFs) harboring either functional or nonfunctional STAT3. SPL silencing in MEFs resulted in an increase in the expression of the Spns2 and a concomitant increase in the extracellular levels of S1P. Moreover, MEFs lacking SPL proliferated faster than those with wild-type SPL and formed tumors when injected subcutaneously in NOD/SCID mice, but only in the presence of wild-type STAT3 and not in its absence. These results combined with our in vivo findings confirmed that SPL silencing renders cells susceptible to cell transformation in a STAT3-dependent manner [147].

Our findings raise additional questions that will require further study. What is the role of SPL contained within other immune cells that are implicated in the development of CAC? Why did we observe an increased number of macrophages in SPL GutKO mice suffering from CAC? This is important, as tumor-associated macrophages are known to play a key role in maintaining the tumor microenvironment suitable for neoplastic transformation [163]. It will also be important to determine how the inflammatory milieu, oxidant stress, diet, and the microbiome affect SPL expression and activity, since gut epithelial SPL and these environmental factors all influence the susceptibility to colitis and CAC.

\section{SPL as a Therapeutic Target for Inflammatory Diseases}

In the past three decades, S1P signaling has been associated with numerous inflammatory diseases such as psoriasis, asthma, rheumatoid arthritis, multiple sclerosis, and inflammatory bowel disease [164]. As novel molecules targeting the sphingolipid pathway components are developed and proven efficacious in preclinical models of inflammatory diseases, as recently reviewed by Park and Im [165], they are moving forward into clinical trials in various disease contexts. In addition to S1P receptor agonist and antagonists [2], SPL inhibitors represent potential treatment options for inflammatory disorders such as multiple sclerosis, rheumatoid arthritis, and inflammatory bowel disease. SPL has a dual role in inflammation. Inhibition of SPL enzyme activity by a pharmacological agent for a short time could inhibit inflammation by blocking the entry of T lymphocytes to the site of injury/damage. In contrast, a sustained decrease in SPL enzyme activity or SPL deficiency will increase S1P levels and thereby may activate NF $\kappa \mathrm{B}$ and STAT3 transcription factors. Both of these transcription factors are known to induce the gene expression of proinflammatory cytokines.

Genetic evidence suggests that partial SPL inhibition may be beneficial in several inflammatory conditions. For example, partial SPL deficiency provides protection in EAE in mice [18]. SPL-deficient mice exhibit reduced migration of $\mathrm{T}$ cells into the CNS [18]. Thus, similar to FTY720, SPL inhibitors could have utility in the treatment of multiple sclerosis. THI, a component of caramel color III, inhibits SPL enzyme activity in vivo but not in vitro. Until recently, the mechanism by which THI inhibits SPL was not clear. Ohtoyo and colleagues revealed that orally delivered THI is metabolized in vivo by the gut microbiome [166]. They showed further that key metabolite of THI, which they synthesized and named A6770, is phosphorylated by cells and inhibits SPL in vitro and in vivo [166]. We have confirmed the activity of A6770 as an SPL inhibitor in vitro, as well as the loss of THImediated lymphopenia by pretreatment with antibiotics to deplete the gut microbiome (our unpublished observations). Lexicon Pharmaceutical Inc. has developed several inhibitors of SPL including LX2931, LX2932, and LX3305. These compounds, which are THI analogs, were shown to inhibit inflammatory responses in animal models of rheumatoid arthritis, multiple sclerosis, and transplantation [167]. Unfortunately, LX3305 did not show promising results in phase II clinical trial in rheumatoid arthritis patients receiving methotrexate [168]. However, considering that methotrexate is an antibiotic that interferes with bacteria's ability to synthesize folate, it is likely that this drug combination abrogated the impact of LX3305 on SPL and, consequently, on inflammation.

Several other examples of the impact of SPL inhibition on inflammation are instructive. A mouse model of cystic fibrosis exhibited reduced levels of S1P in the lungs. Oral treatment of these mice with LX2931 raised S1P levels in the tissues and reduced lung inflammation by decreasing the levels of inflammatory cytokines [80]. Sepsis is a systemic inflammatory response to pathogens and a leading cause of hospital-related mortality worldwide. Administration of 4-deoxypyridoxine (DOP), a nonselective inhibitor of SPL, ameliorated morbidity, improved recovery from sepsis in surviving mice, and reduced sepsis-induced hypothermia. Treated mice also showed reduced bacterial burden in the liver, although not in the blood [169]. NK cells have been shown to localize in the medullary region of lymph nodes, and an S1P gradient is required for the proper localization of NK cells to this site [170]. This raises the possibility that SPL could be targeted in cancer for control NK-cell mediated tumor cytotoxicity. In fact, a recent study supports this notion [171]. Complete deficiency of SPL could enhance transformation and cause severe complications similar to those observed in patients with SGPL1 mutations. However, partial SPL inhibition with small molecule inhibitors may be beneficial in certain contexts, especially using short-term or periodic administration.

Identification of SPL as a promising therapeutic target for inflammatory diseases has intensified interest in the identification of small molecules that modulate its activity. To facilitate testing of a large number of compounds, newer high throughput methods are being developed. Kashem et al. developed a high-throughput scintillation proximity assay to screen SPL inhibitors [172]. The major limitation in the 
development of novel SPL inhibitors is the lack of a crystal structure for human SPL. In order to overcome this problem, bacterial SPL from Symbiobacterium thermophilum has been mutated, which would facilitate structure-based designing of novel SPL inhibitors [173]. Recombinant StSPL has been shown to degrade extracellular S1P in vivo [60]. Thus, StSPL may be used as an enzyme replacement therapy for patients with SPL deficiency, suffering from nephrotic syndrome, adrenal insufficiency, and neurological problems, and for patients with fibrotic kidney disease.

\section{Conclusion}

In conclusion, SPL is a ubiquitously expressed enzyme that is essential for maintaining S1P chemotactic gradients that enable $\mathrm{T}$ cell egress and other aspects of hematopoietic cell trafficking. In addition, SPL appears to regulate S1P/STAT3 signaling in the context of intestinal inflammation, colitis, and the development of CAC. In addition to its influence on S1P signaling, SPL generates unique products that exhibit biological activities and control upstream sphingolipid intermediates, since SPL represents the only exit point for the entire sphingolipid degradative pathway. As such, SPL modulation has pleiotropic effects, and mutations in human SGPL1 are associated with a variety of disease states including but not limited to immunodeficiency. Future studies involving SPL-deficient patients and their biological samples and utilizing SPL inhibitors, model organisms, and global, partial, targeted, and inducible mouse models of SPL deficiency will help clarify the role of SPL in physiology, disease, and therapeutics.

\section{Abbreviations}

ABC: ATP-binding cassette

ApoM: Apolipoprotein M

BM: $\quad$ Bone marrow

BMDC: Bone marrow-derived dendritic cells

CAC: Colitis-associated cancer

CMJ: Corticomedullary junction

CNS: Central nervous system

DC: Dendritic cell

DP: Double-positive

DSS: Dextran sodium sulfate

EAE: Experimental autoimmune encephalomyelitis

ER: Endoplasmic reticulum

GRK: G protein-coupled receptor kinase

HSC: Hematopoietic stem cell

IBD: Inflammatory bowel disease

IL: $\quad$ Interleukin

KLF: Krüppel-like factor

KO: Knockout

LPP: Lipid phosphate phosphatase

LPS: Lipopolysaccharide

MAM: Mitochondria-associated membrane

MEF: $\quad$ Mouse embryonic fibroblast

miR: MicroRNA

$\mathrm{NF} \kappa \mathrm{B}$ : Nuclear factor kappa B

NK: $\quad$ Natural killer
PLP: $\quad$ Pyridoxal phosphate

S1P: $\quad$ Sphingosine-1-phosphate

SPL: $\quad$ Sphingosine-1-phosphate lyase

S1PR: Sphingosine-1-phosphate receptor

STAT3: Signal transducer and activator of transcription 3

SphK: Sphingosine kinase

SC: $\quad$ Satellite cell

SRNS: Steroid-resistant nephrotic syndrome

SP: $\quad$ Single-positive

TEC: Thymic epithelial cell

THI: 2-Acetyl-4-(tetrahydroxybutyl) imidazole

TNF: Tumor necrosis factor

TRAF2: Tumor necrosis factor-associated factor 2

Treg: Regulatory T cell.

\section{Conflicts of Interest}

The authors declare that there is no conflict of interest regarding the publication of this paper.

\section{Acknowledgments}

Ashok Kumar acknowledges the financial support received from Science and Engineering Research Board (SERB), Government of India (SB/YS/LS-184/2013), and Madhya Pradesh Council of Science \& Technology, Bhopal (A/RD/ RP-2/2015-16/50). This review was supported by funds from the Swim Across America Foundation and NIH Grant CA129438 (Julie D. Saba).

\section{References}

[1] H. Fyrst and J. D. Saba, "An update on sphingosine-1phosphate and other sphingolipid mediators," Nature Chemical Biology, vol. 6, no. 7, pp. 489-497, 2010.

[2] G. T. Kunkel, M. Maceyka, S. Milstien, and S. Spiegel, "Targeting the sphingosine-1-phosphate axis in cancer, inflammation and beyond," Nature Reviews Drug Discovery, vol. 12, no. 9, pp. 688-702, 2013.

[3] S. Spiegel and S. Milstien, "The outs and the ins of sphingosine-1-phosphate in immunity," Nature Reviews Immunology, vol. 11, no. 6, pp. 403-415, 2011.

[4] W. Stoffel, B. Hellenbroich, and G. Heimann, "Properties and specificities of sphingosine kinase from blood platelets," Hoppe-Seyler's Zeitschrift für Physiologische Chemie, vol. 354, no. 2, pp. 1311-1316, 1973.

[5] X. Tang, M. G. Benesch, and D. N. Brindley, "Lipid phosphate phosphatases and their roles in mammalian physiology and pathology," Journal of Lipid Research, vol. 56, no. 11, pp. 2048-2060, 2015.

[6] H. Le Stunff, C. Peterson, H. Liu, S. Milstien, and S. Spiegel, "Sphingosine-1-phosphate and lipid phosphohydrolases," Biochimica et Biophysica Acta (BBA) - Molecular and Cell Biology of Lipids, vol. 1582, no. 1-3, pp. 8-17, 2002.

[7] J. Zhou and J. D. Saba, "Identification of the first mammalian sphingosine phosphate lyase gene and its functional expression in yeast," Biochemical and Biophysical Research Communications, vol. 242, no. 3, pp. 502-507, 1998.

[8] P. P. Van Veldhoven, S. Gijsbers, G. P. Mannaerts, J. R. Vermeesch, and V. Brys, "Human sphingosine-1-phosphate 
lyase: cDNA cloning, functional expression studies and mapping to chromosome 10q22," Biochimica et Biophysica Acta (BBA) - Molecular and Cell Biology of Lipids, vol. 1487, no. 23, pp. 128-134, 2000.

[9] A. Aguilar and J. D. Saba, "Truth and consequences of sphingosine-1-phosphate lyase," Advances in Biological Regulation, vol. 52, no. 1, pp. 17-30, 2012.

[10] E. Degagne and J. D. Saba, "Slpping fire: Sphingosine-1phosphate signaling as an emerging target in inflammatory bowel disease and colitis-associated cancer," Clinical and Experimental Gastroenterology, vol. 7, pp. 205-214, 2014.

[11] B. Oskouian, P. Sooriyakumaran, A. D. Borowsky et al., "Sphingosine-1-phosphate lyase potentiates apoptosis via p53- and p38-dependent pathways and is down-regulated in colon cancer," Proceedings of the National Academy of Sciences of the United States of America, vol. 103, no. 46, pp. 17384-17389, 2006.

[12] A. Kumar, B. Oskouian, H. Fyrst, M. Zhang, F. Paris, and J. D. Saba, "S1P lyase regulates DNA damage responses through a novel sphingolipid feedback mechanism," Cell Death \& Disease, vol. 2, no. 2, article e119, 2011.

[13] K. C. Loh, W. I. Leong, M. E. Carlson et al., "Sphingosine-1phosphate enhances satellite cell activation in dystrophic muscles through a S1PR2/STAT3 signaling pathway," PLoS One, vol. 7, no. 5, article e37218, 2012.

[14] S. R. Schwab, J. P. Pereira, M. Matloubian, Y. Xu, Y. Huang, and J. G. Cyster, "Lymphocyte sequestration through S1P lyase inhibition and disruption of S1P gradients," Science, vol. 309, no. 5741, pp. 1735-1739, 2005.

[15] P. Bandhuvula, N. Honbo, G. Y. Wang et al., "S1P lyase: a novel therapeutic target for ischemia-reperfusion injury of the heart," American Journal of Physiology Heart and Circulatory Physiology, vol. 300, no. 5, pp. H1753-H1761, 2011.

[16] A. S. de la Garza-Rodea, D. M. Baldwin, B. Oskouian et al., "Sphingosine phosphate lyase regulates myogenic differentiation via S1P receptor-mediated effects on myogenic microRNA expression," The FASEB Journal, vol. 28, no. 1, pp. 506-519, 2014.

[17] G. S. Smith, A. Kumar, and J. D. Saba, "Sphingosine phosphate lyase regulates murine embryonic stem cell proliferation and pluripotency through an S1P2/STAT3 signaling pathway," Biomolecules, vol. 3, no. 3, pp. 351-368, 2013.

[18] A. Billich, T. Baumruker, C. Beerli et al., "Partial deficiency of sphingosine-1-phosphate lyase confers protection in experimental autoimmune encephalomyelitis," PLoS One, vol. 8, no. 3, article e59630, 2013.

[19] M. Bot, P. P. van Veldhoven, S. C. A. de Jager et al., "Hematopoietic sphingosine 1-phosphate lyase deficiency decreases atherosclerotic lesion development in LDL-receptor deficient mice," PLoS One, vol. 8, no. 5, article e63360, 2013.

[20] A. R. Janecke, R. Xu, E. Steichen-Gersdorf et al., "Deficiency of the sphingosine-1-phosphate lyase SGPL1 is associated with congenital nephrotic syndrome and congenital adrenal calcifications," Human Mutation, vol. 38, no. 4, pp. 365372, 2017.

[21] R. Prasad, I. Hadjidemetriou, A. Maharaj et al., "Sphingosine1-phosphate lyase mutations cause primary adrenal insufficiency and steroid-resistant nephrotic syndrome," Journal of Clinical Investigation, vol. 127, no. 3, pp. 942-953, 2017.

[22] K. Zhang, J. M. Pompey, F. F. Hsu et al., "Redirection of sphingolipid metabolism toward de novo synthesis of ethanolamine in Leishmania," The EMBO Journal, vol. 26, no. 4, pp. 1094-1104, 2007.

[23] D. L. Ebenezer, P. Fu, V. Suryadevara, Y. Zhao, and V. Natarajan, "Epigenetic regulation of pro-inflammatory cytokine secretion by sphingosine 1-phosphate (S1P) in acute lung injury: role of S1P lyase," Advances in Biological Regulation, vol. 63, pp. 156-166, 2017.

[24] A. Kumar, H. S. Byun, R. Bittman, and J. D. Saba, "The sphingolipid degradation product trans-2-hexadecenal induces cytoskeletal reorganization and apoptosis in a JNdependent manner," Cellular Signalling, vol. 23, no. 7, pp. 1144-1152, 2011.

[25] J. E. Chipuk, G. P. McStay, A. Bharti et al., "Sphingolipid metabolism cooperates with BAK and BAX to promote the mitochondrial pathway of apoptosis," Cell, vol. 148, no. 5, pp. 988-1000, 2012.

[26] M. Matloubian, C. G. Lo, G. Cinamon et al., "Lymphocyte egress from thymus and peripheral lymphoid organs is dependent on S1P receptor 1," Nature, vol. 427, no. 6972, pp. 355-360, 2004.

[27] R. L. Proia and T. Hla, "Emerging biology of sphingosine1-phosphate: its role in pathogenesis and therapy," Journal of Clinical Investigation, vol. 125, no. 4, pp. 1379-1387, 2015.

[28] V. Brinkmann, A. Billich, T. Baumruker et al., "Fingolimod (FTY720): discovery and development of an oral drug to treat multiple sclerosis," Nature Reviews Drug Discovery, vol. 9, no. 11, pp. 883-897, 2010.

[29] H. C. Tsai and M. H. Han, "Sphingosine-1-phosphate (S1P) and S1P signaling pathway: therapeutic targets in autoimmunity and inflammation," Drugs, vol. 76, no. 11, pp. 10671079, 2016.

[30] J. Rivera, R. L. Proia, and A. Olivera, "The alliance of sphingosine-1-phosphate and its receptors in immunity," Nature Reviews Immunology, vol. 8, no. 10, pp. 753-763, 2008.

[31] A. Kumar and J. D. Saba, "Regulation of immune cell migration by sphingosine-1-phosphate," Cellular and Molecular Biology, vol. 61, no. 2, 2015.

[32] F. Balkwill and A. Mantovani, "Inflammation and cancer: back to Virchow?," The Lancet, vol. 357, no. 9255, pp. 539545, 2001.

[33] B. Yang, H. Kang, A. Fung, H. Zhao, T. Wang, and D. Ma, "The role of interleukin 17 in tumour proliferation, angiogenesis, and metastasis," Mediators of Inflammation, vol. 2014, Article ID 623759, 12 pages, 2014.

[34] A. J. Templeton, M. G. McNamara, B. Šeruga et al., "Prognostic role of neutrophil-to-lymphocyte ratio in solid tumors: a systematic review and meta-analysis," Journal of the National Cancer Institute, vol. 106, no. 6, article dju124, 2014.

[35] S. Spiegel and S. Milstien, "Sphingosine-1-phosphate: an enigmatic signalling lipid," Nature Reviews Molecular Cell Biology, vol. 4, no. 5, pp. 397-407, 2003.

[36] N. Igarashi, T. Okada, S. Hayashi, T. Fujita, S. Jahangeer, and S.i. Nakamura, "Sphingosine kinase 2 is a nuclear protein and inhibits DNA synthesis," The Journal of Biological Chemistry, vol. 278, no. 47, pp. 46832-46839, 2003.

[37] H. Le Stunff, C. Peterson, R. Thornton, S. Milstien, S. M. Mandala, and S. Spiegel, "Characterization of murine sphingosine-1-phosphate phosphohydrolase," Journal of Biological Chemistry, vol. 277, no. 11, pp. 8920-8927, 2002. 
[38] C. Ogawa, A. Kihara, M. Gokoh, and Y. Igarashi, "Identification and characterization of a novel human sphingosine-1phosphate phosphohydrolase, hSPP2," Journal of Biological Chemistry, vol. 278, no. 2, pp. 1268-1272, 2003.

[39] R. Jasinska, Q. X. Zhang, C. Pilquil et al., "Lipid phosphate phosphohydrolase-1 degrades exogenous glycerolipid and sphingolipid phosphate esters," Biochemical Journal, vol. 340, no. 3, pp. 677-686, 1999.

[40] D. N. Brindley, D. English, C. Pilquil, K. Buri, and Z. C. Ling, "Lipid phosphate phosphatases regulate signal transduction through glycerolipids and sphingolipids," Biochimica et Biophysica Acta (BBA) - Molecular and Cell Biology of Lipids, vol. 1582, no. 1-3, pp. 33-44, 2002.

[41] J. L. Tanyi, A. J. Morris, J. K. Wolf et al., "The human lipid phosphate phosphatase-3 decreases the growth, survival, and tumorigenesis of ovarian cancer cells: validation of the lysophosphatidic acid signaling cascade as a target for therapy in ovarian cancer," Cancer Research, vol. 63, no. 5, pp. 1073-1082, 2003.

[42] N. J. Pyne and S. Pyne, "Sphingosine 1-phosphate and cancer," Nature Reviews Cancer, vol. 10, no. 7, pp. 489-503, 2010.

[43] D. Mechtcheriakova, A. Wlachos, J. Sobanov et al., "FTY720phosphate is dephosphorylated by lipid phosphate phosphatase 3," FEBS Letters, vol. 581, no. 16, pp. 3063-3068, 2007.

[44] X. Tang, Y. Y. Zhao, J. Dewald, J. M. Curtis, and D. N. Brindley, "Tetracyclines increase lipid phosphate phosphatase expression on plasma membranes and turnover of plasma lysophosphatidate," Journal of Lipid Research, vol. 57, no. 4, pp. 597-606, 2016.

[45] B. Breart, W. D. Ramos-Perez, A. Mendoza et al., "Lipid phosphate phosphatase 3 enables efficient thymic egress," The Journal of Experimental Medicine, vol. 208, no. 6, pp. 1267-1278, 2011.

[46] F. Bourquin, G. Capitani, and M. G. Grutter, "PLP-dependent enzymes as entry and exit gates of sphingolipid metabolism," Protein Science, vol. 20, no. 9, pp. 1492-1508, 2011.

[47] W. Stoffel, D. LeKim, and G. Sticht, "Distribution and properties of dihydrosphingosine-1-phosphate aldolase (sphinganine-1-phosphate alkanal-lyase)," Hoppe-Seyler's Zeitschrift für Physiologische Chemie, vol. 350, no. 2, pp. 1233-1241, 1969.

[48] W. Stoffel and M. Grol, "Chemistry and biochemistry of 1-desoxysphinganine 1-phosphonate (dihydrosphingosine1-phosphonate)," Chemistry and Physics of Lipids, vol. 13, no. 4, pp. 372-388, 1974.

[49] J. D. Saba, F. Nara, A. Bielawska, S. Garrett, and Y. A. Hannun, "The BST1 gene of Saccharomyces cerevisiae is the sphingosine-1-phosphate lyase," Journal of Biological Chemistry, vol. 272, no. 42, pp. 26087-26090, 1997.

[50] D. R. Herr, H. Fyrst, V. Phan et al., "Sply regulation of sphingolipid signaling molecules is essential for Drosophila development," Development, vol. 130, no. 11, pp. 2443-2453, 2003.

[51] J. Mendel, K. Heinecke, H. Fyrst, and J. D. Saba, "Sphingosine phosphate lyase expression is essential for normal development in Caenorhabditis elegans," Journal of Biological Chemistry, vol. 278, no. 25, pp. 22341-22349, 2003.

[52] G. Li, C. Foote, S. Alexander, and H. Alexander, "Sphingosine-1-phosphate lyase has a central role in the development of Dictyostelium discoideum," Development, vol. 128, no. 18, pp. 3473-3483, 2001.
[53] M. Rolando, P. Escoll, T. Nora et al., "Legionella pneumophila S1P-lyase targets host sphingolipid metabolism and restrains autophagy," Proceedings of the National Academy of Sciences of the United States of America, vol. 113, no. 7, pp. 1901-1906, 2016.

[54] M. M. Adada, K. A. Orr-Gandy, A. J. Snider et al., "Sphingosine kinase 1 regulates tumor necrosis factor-mediated RANTES induction through p38 mitogen-activated protein kinase but independently of nuclear factor $\kappa \mathrm{B}$ activation," Journal of Biological Chemistry, vol. 288, no. 38, pp. 2766727679, 2013.

[55] H. Zhang, J. Zhai, J. Mo, D. Li, and F. Song, "Overexpression of rice sphingosine-1-phoshpate lyase gene OsSPL1 in transgenic tobacco reduces salt and oxidative stress tolerance," Journal of Integrative Plant Biology, vol. 54, no. 9, pp. 652662, 2012.

[56] F. Bourquin, H. Riezman, G. Capitani, and M. G. Grütter, "Structure and function of sphingosine-1-phosphate lyase, a key enzyme of sphingolipid metabolism," Structure, vol. 18, no. 8, pp. 1054-1065, 2010.

[57] H. Fyrst and J. D. Saba, "Sphingosine-1-phosphate lyase in development and disease: sphingolipid metabolism takes flight," Biochimica et Biophysica Acta (BBA) - Molecular and Cell Biology of Lipids, vol. 1781, no. 9, pp. 448-458, 2008.

[58] D. Mukhopadhyay, K. S. Howell, H. Riezman, and G. Capitani, "Identifying key residues of sphinganine-1phosphate lyase for function in vivo and in vitro," Journal of Biological Chemistry, vol. 283, no. 29, pp. 20159-20169, 2008.

[59] M. Ikeda, A. Kihara, and Y. Igarashi, "Sphingosine-1phosphate lyase SPL is an endoplasmic reticulum-resident, integral membrane protein with the pyridoxal $5^{\prime}$-phosphate binding domain exposed to the cytosol," Biochemical and Biophysical Research Communications, vol. 325, no. 1, pp. 338-343, 2004.

[60] A. Huwiler, F. Bourquin, N. Kotelevets et al., "A prokaryotic S1P lyase degrades extracellular S1P in vitro and in vivo: implication for treating hyperproliferative disorders," PLoS One, vol. 6, no. 8, article e22436, 2011.

[61] A. D. Borowsky, P. Bandhuvula, A. Kumar et al., "Sphingosine-1-phosphate lyase expression in embryonic and adult murine tissues," Journal of Lipid Research, vol. 53, no. 9, pp. 1920-1931, 2012.

[62] K. Ito, Y. Anada, M. Tani et al., "Lack of sphingosine 1phosphate-degrading enzymes in erythrocytes," Biochemical and Biophysical Research Communications, vol. 357, no. 1, pp. 212-217, 2007.

[63] Y. Yatomi, Y. Igarashi, L. Yang et al., "Sphingosine 1phosphate, a bioactive sphingolipid abundantly stored in platelets, is a normal constituent of human plasma and serum," Journal of Biochemistry, vol. 121, no. 5, pp. 969973, 1997.

[64] S. Newbigging, M. Zhang, and J. D. Saba, "Immunohistochemical analysis of sphingosine phosphate lyase expression during murine development," Gene Expression Patterns, vol. 13, no. 1-2, pp. 21-29, 2013.

[65] M. B. Genter, P. P. van Veldhoven, A. G. Jegga et al., "Microarray-based discovery of highly expressed olfactory mucosal genes: potential roles in the various functions of the olfactory system," Physiological Genomics, vol. 16, no. 1, pp. 67-81, 2003. 
[66] A. Kumar and J. D. Saba, "Lyase to live by: sphingosine phosphate lyase as a therapeutic target," Expert Opinion on Therapeutic Targets, vol. 13, no. 8, pp. 1013-1025, 2009.

[67] H. Vienken, N. Mabrouki, K. Grabau et al., "Characterization of cholesterol homeostasis in sphingosine-1-phosphate lyase-deficient fibroblasts reveals a Niemann-Pick disease type C-like phenotype with enhanced lysosomal $\mathrm{Ca}^{2+}$ storage," Scientific Reports, vol. 7, article 43575, 2017.

[68] J. Schmahl, C. S. Raymond, and P. Soriano, "PDGF signaling specificity is mediated through multiple immediate early genes," Nature Genetics, vol. 39, no. 1, pp. 52-60, 2007.

[69] M. Bektas, M. L. Allende, B. G. Lee et al., "Sphingosine 1phosphate lyase deficiency disrupts lipid homeostasis in liver," The Journal of Biological Chemistry, vol. 285, no. 14, pp. 10880-10889, 2010.

[70] P. Vogel, M. S. Donoviel, R. Read et al., "Incomplete inhibition of sphingosine 1-phosphate lyase modulates immune system function yet prevents early lethality and nonlymphoid lesions," PLoS One, vol. 4, no. 1, article e4112, 2009.

[71] J. Min, A. L. Stegner, H. Alexander, and S. Alexander, "Overexpression of sphingosine-1-phosphate lyase or inhibition of sphingosine kinase in Dictyostelium discoideum results in a selective increase in sensitivity to platinum-based chemotherapy drugs," Eukaryotic Cell, vol. 3, no. 3, pp. 795-805, 2004.

[72] K. Ihlefeld, H. Vienken, R. F. Claas et al., "Upregulation of $\mathrm{ABC}$ transporters contributes to chemoresistance of sphingosine 1-phosphate lyase-deficient fibroblasts," Journal of Lipid Research, vol. 56, no. 1, pp. 60-69, 2015.

[73] K. Matula, E. Collie-Duguid, G. Murray et al., "Regulation of cellular sphingosine-1-phosphate by sphingosine kinase 1 and sphingosine-1-phopshate lyase determines chemotherapy resistance in gastroesophageal cancer," BMC Cancer, vol. 15, no. 1, p. 762, 2015.

[74] L. Brizuela, I. Ader, C. Mazerolles, M. Bocquet, B. Malavaud, and O. Cuvillier, "First evidence of sphingosine 1-phosphate lyase protein expression and activity downregulation in human neoplasm: implication for resistance to therapeutics in prostate cancer," Molecular Cancer Therapeutics, vol. 11, no. 9, pp. 1841-1851, 2012.

[75] S. Colie, P. P. van Veldhoven, B. Kedjouar et al., "Disruption of sphingosine 1-phosphate lyase confers resistance to chemotherapy and promotes oncogenesis through $\mathrm{Bcl}-2 / \mathrm{Bcl}-\mathrm{xL}$ upregulation," Cancer Research, vol. 69, no. 24, pp. 93469353, 2009.

[76] S. N. Patmanathan, S. P. Johnson, S. L. Lai et al., "Aberrant expression of the S1P regulating enzymes, SPHK1 and SGPL1, contributes to a migratory phenotype in OSCC mediated through S1PR2," Scientific Reports, vol. 6, no. 1, article 25650, 2016.

[77] K. Hibbs, K. M. Skubitz, S. E. Pambuccian et al., "Differential gene expression in ovarian carcinoma: identification of potential biomarkers," The American Journal of Pathology, vol. 165, no. 2, pp. 397-414, 2004.

[78] B. Uranbileg, H. Ikeda, M. Kurano et al., "Increased mRNA levels of sphingosine kinases and S1P lyase and reduced levels of S1P were observed in hepatocellular carcinoma in association with poorer differentiation and earlier recurrence," PLoS One, vol. 11, no. 2, article e0149462, 2016.

[79] S. Vishwakarma, R. Agarwal, S. K. Goel et al., "Altered expression of sphingosine-1-phosphate metabolizing enzymes in oral cancer correlate with clinicopathological attributes," Cancer Investigation, vol. 35, no. 2, pp. 139-141, 2017.
[80] M. Veltman, M. Stolarczyk, D. Radzioch et al., "Correction of lung inflammation in a F508del CFTR murine cystic fibrosis model by the sphingosine-1-phosphate lyase inhibitor LX2931," American Journal of Physiology. Lung Cellular and Molecular Physiology, vol. 311, no. 5, pp. L1000-L1014, 2016.

[81] L. S. Huang, E. V. Berdyshev, J. T. Tran et al., "Sphingosine-1phosphate lyase is an endogenous suppressor of pulmonary fibrosis: role of S1P signalling and autophagy," Thorax, vol. 70, no. 12, pp. 1138-1148, 2015.

[82] Y. Zhao, I. A. Gorshkova, E. Berdyshev et al., "Protection of LPS-induced murine acute lung injury by sphingosine-1phosphate lyase suppression," American Journal of Respiratory Cell and Molecular Biology, vol. 45, no. 2, pp. 426-435, 2011.

[83] M. Rolando, P. Escoll, and C. Buchrieser, "Legionella pneumophila restrains autophagy by modulating the host's sphingolipid metabolism," Autophagy, vol. 12, no. 6, pp. 1053-1054, 2016.

[84] C. J. McLean, J. Marles-Wright, R. Custodio et al., "Characterization of homologous sphingosine-1-phosphate lyase isoforms in the bacterial pathogen Burkholderia pseudomallei," Journal of Lipid Research, vol. 58, no. 1, pp. 137-150, 2017.

[85] R. Custodio, C. J. McLean, A. E. Scott et al., "Characterization of secreted sphingosine-1-phosphate lyases required for virulence and intracellular survival of Burkholderia pseudomallei," Molecular Microbiology, vol. 102, no. 6, pp. 1004-1019, 2016.

[86] S. Lovric, S. Goncalves, H. Y. Gee et al., "Mutations in sphingosine-1-phosphate lyase cause nephrosis with ichthyosis and adrenal insufficiency," Journal of Clinical Investigation, vol. 127, no. 3, pp. 912-928, 2017.

[87] D. Atkinson, J. Nikodinovic Glumac, B. Asselbergh et al., "Sphingosine 1-phosphate lyase deficiency causes CharcotMarie-Tooth neuropathy," Neurology, vol. 88, no. 6, pp. 533-542, 2017.

[88] D. N. Mitroi, A. U. Deutschmann, M. Raucamp et al., "Sphingosine 1-phosphate lyase ablation disrupts presynaptic architecture and function via an ubiquitin- proteasome mediated mechanism," Scientific Reports, vol. 6, no. 1, article 37064, 2016.

[89] D. N. Mitroi, I. Karunakaran, M. Gräler et al., "SGPL1 (sphingosine phosphate lyase 1) modulates neuronal autophagy via phosphatidylethanolamine production," Autophagy, vol. 13, no. 5, pp. 885-899, 2017.

[90] Y. Kariya, A. Kihara, M. Ikeda et al., "Products by the sphingosine kinase/sphingosine 1-phosphate (S1P) lyase pathway but not S1P stimulate mitogenesis," Genes to Cells, vol. 10, no. 6, pp. 605-615, 2005.

[91] P. Upadhyaya, A. Kumar, H. S. Byun, R. Bittman, J. D. Saba, and S. S. Hecht, "The sphingolipid degradation product trans-2-hexadecenal forms adducts with DNA," Biochemical and Biophysical Research Communications, vol. 424, no. 1, pp. 18-21, 2012.

[92] F. Schumacher, C. Neuber, H. Finke et al., "The sphingosine 1-phosphate break-down product (2E)-hexadecenal forms protein adducts and glutathione conjugates in vitro," Journal of Lipid Research, vol. 58, no. 8, pp. 1648-1660, 2017.

[93] C. N. Jenne, A. Enders, R. Rivera et al., "T-bet-dependent S1P5 expression in NK cells promotes egress from lymph nodes and bone marrow," The Journal of Experimental Medicine, vol. 206, no. 11, pp. 2469-2481, 2009. 
[94] P. S. Jolly, M. Bektas, A. Olivera et al., "Transactivation of sphingosine-1-phosphate receptors by FceRI triggering is required for normal mast cell degranulation and chemotaxis," The Journal of Experimental Medicine, vol. 199, no. 7, pp. 959-970, 2004.

[95] K. Konig, L. Diehl, U. Rommerscheidt-Fuss et al., "Four-anda-half LIM domain protein 2 is a novel regulator of sphingosine 1-phosphate receptor 1 in CCL19-induced dendritic cell migration," Journal of Immunology, vol. 185, no. 3, pp. 14661475, 2010.

[96] A. Rathinasamy, N. Czeloth, O. Pabst, R. Forster, and G. Bernhardt, "The origin and maturity of dendritic cells determine the pattern of sphingosine 1-phosphate receptors expressed and required for efficient migration," Journal of Immunology, vol. 185, no. 7, pp. 4072-4081, 2010.

[97] S. R. Schwab and J. G. Cyster, "Finding a way out: lymphocyte egress from lymphoid organs," Nature Immunology, vol. 8, no. 12, pp. 1295-1301, 2007.

[98] M. A. Zachariah and J. G. Cyster, "Neural crest-derived pericytes promote egress of mature thymocytes at the corticomedullary junction," Science, vol. 328, no. 5982, pp. 1129-1135, 2010.

[99] L. Zhang, M. Orban, M. Lorenz et al., "A novel role of sphingosine 1-phosphate receptor S1pr1 in mouse thrombopoiesis," The Journal of Experimental Medicine, vol. 209, no. 12, pp. 2165-2181, 2012.

[100] R. K. Sinha, C. Park, I. Y. Hwang, M. D. Davis, and J. H. Kehrl, "B lymphocytes exit lymph nodes through cortical lymphatic sinusoids by a mechanism independent of sphingosine-1-phosphate-mediated chemotaxis," Immunity, vol. 30, no. 3, pp. 434-446, 2009.

[101] M. E. Ogle, C. E. Olingy, A. O. Awojoodu et al., "Sphingosine1-phosphate receptor-3 supports hematopoietic stem and progenitor cell residence within the bone marrow niche," Stem Cells, vol. 35, no. 4, pp. 1040-1052, 2017.

[102] M. Adamiak, S. Borkowska, M. Wysoczynski et al., "Evidence for the involvement of sphingosine-1-phosphate in the homing and engraftment of hematopoietic stem cells to bone marrow," Oncotarget, vol. 6, no. 22, pp. 1881918828, 2015

[103] M. Chimen, H. M. McGettrick, B. Apta et al., "Homeostatic regulation of $\mathrm{T}$ cell trafficking by a $\mathrm{B}$ cell-derived peptide is impaired in autoimmune and chronic inflammatory disease," Nature Medicine, vol. 21, no. 5, pp. 467-475, 2015.

[104] T. Nitta, S. Murata, T. Ueno, K. Tanaka, and Y. Takahama, "Thymic microenvironments for T-cell repertoire formation," Advances in Immunology, vol. 99, pp. 59-94, 2008.

[105] M. A. Weinreich and K. A. Hogquist, "Thymic emigration: when and how T cells leave home," Journal of Immunology, vol. 181, no. 4, pp. 2265-2270, 2008.

[106] C. M. Carlson, B. T. Endrizzi, J. Wu et al., "Kruppel-like factor 2 regulates thymocyte and T-cell migration," Nature, vol. 442, no. 7100, pp. 299-302, 2006.

[107] A. Bai, H. Hu, M. Yeung, and J. Chen, "Kruppel-like factor 2 controls $\mathrm{T}$ cell trafficking by activating L-selectin (CD62L) and sphingosine-1-phosphate receptor 1 transcription," Journal of Immunology, vol. 178, no. 12, pp. 7632-7639, 2007.

[108] Y. M. Kerdiles, D. R. Beisner, R. Tinoco et al., "Foxo1 links homing and survival of naive T cells by regulating L-selectin, CCR7 and interleukin 7 receptor," Nature Immunology, vol. 10, no. 2, pp. 176-184, 2009.
[109] C. N. Skon, J. Y. Lee, K. G. Anderson, D. Masopust, K. A. Hogquist, and S. C. Jameson, "Transcriptional downregulation of S1pr1 is required for the establishment of resident memory CD8 ${ }^{+}$T cells," Nature Immunology, vol. 14, no. 12, pp. 1285-1293, 2013.

[110] J. G. Cyster and S. R. Schwab, "Sphingosine-1-phosphate and lymphocyte egress from lymphoid organs," Annual Review of Immunology, vol. 30, no. 1, pp. 69-94, 2012.

[111] Y. Dong, X. Du, J. Ye et al., "A cell-intrinsic role for Mst1 in regulating thymocyte egress," The Journal of Immunology, vol. 183, no. 6, pp. 3865-3872, 2009.

[112] F. Mou, M. Praskova, F. Xia et al., "The Mst1 and Mst2 kinases control activation of rho family GTPases and thymic egress of mature thymocytes," The Journal of Experimental Medicine, vol. 209, no. 4, pp. 741-759, 2012.

[113] M. L. Allende, J. L. Dreier, S. Mandala, and R. L. Proia, "Expression of the sphingosine 1-phosphate receptor, S1P1, on T-cells controls thymic emigration," Journal of Biological Chemistry, vol. 279, no. 15, pp. 15396-15401, 2004.

[114] L. R. Shiow, D. B. Rosen, N. Brdičková et al., "CD69 acts downstream of interferon-?/? to inhibit S1P1 and lymphocyte egress from lymphoid organs," Nature, vol. 440, no. 7083, pp. 540-544, 2006.

[115] A. J. Bankovich, L. R. Shiow, and J. G. Cyster, "CD69 suppresses sphingosine 1-phosophate receptor-1 (S1P1) function through interaction with membrane helix 4," The Journal of Biological Chemistry, vol. 285, no. 29, pp. 22328-22337, 2010.

[116] H. Rosen, C. Alfonso, C. D. Surh, and M. G. McHeyzerWilliams, "Rapid induction of medullary thymocyte phenotypic maturation and egress inhibition by nanomolar sphingosine 1-phosphate receptor agonist," Proceedings of the National Academy of Sciences of the United States of America, vol. 100, no. 19, pp. 10907-10912, 2003.

[117] K. R. Watterson, E. Johnston, C. Chalmers et al., "Dual regulation of EDG1/S1P(1) receptor phosphorylation and internalization by protein kinase $\mathrm{C}$ and G-protein-coupled receptor kinase 2," The Journal of Biological Chemistry, vol. 277, no. 8, pp. 5767-5777, 2002.

[118] T. I. Arnon, Y. Xu, C. Lo et al., "GRK2-dependent S1PR1 desensitization is required for lymphocytes to overcome their attraction to blood," Science, vol. 333, no. 6051, pp. 18981903, 2011.

[119] C. H. Liu, S. Thangada, M. J. Lee, J. R. van Brocklyn, S. Spiegel, and T. Hla, "Ligand-induced trafficking of the sphingosine-1-phosphate receptor EDG-1," Molecular Biology of the Cell, vol. 10, no. 4, pp. 1179-1190, 1999.

[120] M. L. Oo, S. H. Chang, S. Thangada et al., "Engagement of $\mathrm{S}_{\mathrm{P}} \mathrm{P}_{1}$-degradative mechanisms leads to vascular leak in mice," Journal of Clinical Investigation, vol. 121, no. 6, pp. 22902300, 2011.

[121] S. S. Ferguson, "Evolving concepts in G protein-coupled receptor endocytosis: the role in receptor desensitization and signaling," Pharmacological Reviews, vol. 53, no. 1, pp. 1-24, 2001.

[122] A. Sorkin and M. von Zastrow, "Endocytosis and signalling: intertwining molecular networks," Nature Reviews Molecular Cell Biology, vol. 10, no. 9, pp. 609-622, 2009.

[123] T. Willinger, S. M. Ferguson, J. P. Pereira, P. de Camilli, and R. A. Flavell, "Dynamin 2-dependent endocytosis is required for sustained S1PR1 signaling," The Journal of Experimental Medicine, vol. 211, no. 4, pp. 685-700, 2014. 
[124] J. Gatfield, L. Monnier, R. Studer, M. H. Bolli, B. Steiner, and O. Nayler, "Sphingosine-1-phosphate (S1P) displays sustained S1P1 receptor agonism and signaling through S1P lyase-dependent receptor recycling," Cellular Signalling, vol. 26, no. 7, pp. 1576-1588, 2014.

[125] M. Ksiazek, M. Chacińska, A. Chabowski, and M. Baranowski, "Sources, metabolism, and regulation of circulating sphingosine-1-phosphate," Journal of Lipid Research, vol. 56, no. 7, pp. 1271-1281, 2015.

[126] Y. Yatomi, "Plasma sphingosine 1-phosphate metabolism and analysis," Biochimica et Biophysica Acta (BBA) - General Subjects, vol. 1780, no. 3, pp. 606-611, 2008.

[127] R. Pappu, S. R. Schwab, I. Cornelissen et al., "Promotion of lymphocyte egress into blood and lymph by distinct sources of sphingosine-1-phosphate," Science, vol. 316, no. 5822, pp. 295-298, 2007.

[128] T. Nishi, N. Kobayashi, Y. Hisano, A. Kawahara, and A. Yamaguchi, "Molecular and physiological functions of sphingosine 1-phosphate transporters," Biochimica et Biophysica Acta (BBA) - Molecular and Cell Biology of Lipids, vol. 1841, no. 5, pp. 759-765, 2014.

[129] P. Mitra, C. A. Oskeritzian, S. G. Payne, M. A. Beaven, S. Milstien, and S. Spiegel, "Role of ABCC1 in export of sphingosine-1-phosphate from mast cells," Proceedings of the National Academy of Sciences of the United States of America, vol. 103, no. 44, pp. 16394-16399, 2006.

[130] N. Kobayashi, T. Nishi, T. Hirata et al., "Sphingosine 1phosphate is released from the cytosol of rat platelets in a carrier-mediated manner," Journal of Lipid Research, vol. 47, no. 3, pp. 614-621, 2006.

[131] A. Mendoza, B. Bréart, W. D. Ramos-Perez et al., "The transporter Spns2 is required for secretion of lymph but not plasma sphingosine-1-phosphate," Cell Reports, vol. 2, no. 5, pp. 1104-1110, 2012.

[132] M. Nagahashi, E. Y. Kim, A. Yamada et al., "Spns2, a transporter of phosphorylated sphingoid bases, regulates their blood and lymph levels, and the lymphatic network," The FASEB Journal, vol. 27, no. 3, pp. 10011011, 2013.

[133] A. Kawahara, T. Nishi, Y. Hisano, H. Fukui, A. Yamaguchi, and N. Mochizuki, "The sphingolipid transporter spns2 functions in migration of zebrafish myocardial precursors," Science, vol. 323, no. 5913, pp. 524-527, 2009.

[134] S. Fukuhara, S. Simmons, S. Kawamura et al., "The sphingosine-1-phosphate transporter Spns2 expressed on endothelial cells regulates lymphocyte trafficking in mice," Journal of Clinical Investigation, vol. 122, no. 4, pp. 14161426, 2012.

[135] A. Nijnik, S. Clare, C. Hale et al., "The role of sphingosine-1phosphate transporter Spns2 in immune system function," The Journal of Immunology, vol. 189, no. 1, pp. 102-111, 2012.

[136] C. Christoffersen, H. Obinata, S. B. Kumaraswamy et al., "Endothelium-protective sphingosine-1-phosphate provided by HDL-associated apolipoprotein M," Proceedings of the National Academy of Sciences of the United States of America, vol. 108, no. 23, pp. 9613-9618, 2011.

[137] F. Poti, M. Simoni, and J. R. Nofer, "Atheroprotective role of high-density lipoprotein (HDL)-associated sphingosine-1phosphate (S1P)," Cardiovascular Research, vol. 103, no. 3, pp. 395-404, 2014.
[138] J. G. Cyster, “Chemokines, sphingosine-1-phosphate, and cell migration in secondary lymphoid organs," Annual Review of Immunology, vol. 23, no. 1, pp. 127-159, 2005.

[139] H. Rosen and E. J. Goetzl, "Sphingosine 1-phosphate and its receptors: an autocrine and paracrine network," Nature Reviews Immunology, vol. 5, no. 7, pp. 560-570, 2005.

[140] J. Zamora-Pineda, A. Kumar, J. H. Suh, M. Zhang, and J. D. Saba, "Dendritic cell sphingosine-1-phosphate lyase regulates thymic egress," The Journal of Experimental Medicine, vol. 213, no. 12, pp. 2773-2791, 2016.

[141] Y. Maeda, H. Yagi, K. Takemoto et al., "S1P lyase in thymic perivascular spaces promotes egress of mature thymocytes via up-regulation of S1P receptor 1," International Immunology, vol. 26, no. 5, pp. 245-255, 2014.

[142] L. Wu and K. Shortman, "Heterogeneity of thymic dendritic cells," Seminars in Immunology, vol. 17, no. 4, pp. 304-312, 2005.

[143] M. Rieck, C. Kremser, K. Jobin et al., "Ceramide synthase 2 facilitates S1P-dependent egress of thymocytes into the circulation in mice," European Journal of Immunology, vol. 47, no. 4, pp. 677-684, 2017.

[144] W. D. Ramos-Perez, V. Fang, D. Escalante-Alcalde, M. Cammer, and S. R. Schwab, "A map of the distribution of sphingosine 1-phosphate in the spleen," Nature Immunology, vol. 16, no. 12, pp. 1245-1252, 2015.

[145] S. E. Alvarez, K. B. Harikumar, N. C. Hait et al., "Sphingosine-1-phosphate is a missing cofactor for the E3 ubiquitin ligase TRAF2," Nature, vol. 465, no. 7301, pp. 1084-1088, 2010.

[146] T. Terashita, K. Kobayashi, T. Nagano et al., “Administration of JTE013 abrogates experimental asthma by regulating proinflammatory cytokine production from bronchial epithelial cells," Respiratory Research, vol. 17, no. 1, p. 146, 2016.

[147] E. Degagne, A. Pandurangan, P. Bandhuvula et al., "Sphingosine-1-phosphate lyase downregulation promotes colon carcinogenesis through STAT3-activated microRNAs," Journal of Clinical Investigation, vol. 124, no. 12, pp. 5368-5384, 2014.

[148] A. V. Nguyen, Y. Y. Wu, Q. Liu et al., "STAT3 in epithelial cells regulates inflammation and tumor progression to malignant state in colon," Neoplasia, vol. 15, no. 9, pp. 998-1008, 2013.

[149] S. Muller, J. Lory, N. Corazza et al., "Activated CD4+ and CD8+ cytotoxic cells are present in increased numbers in the intestinal mucosa from patients with active inflammatory bowel disease," The American Journal of Pathology, vol. 152, no. 1, pp. 261-268, 1998.

[150] T. R. Mempel, M. J. Pittet, K. Khazaie et al., "Regulatory T cells reversibly suppress cytotoxic $\mathrm{T}$ cell function independent of effector differentiation," Immunity, vol. 25, no. 1, pp. 129-141, 2006.

[151] M. L. Allende, M. Bektas, B. G. Lee et al., "Sphingosine-1phosphate lyase deficiency produces a pro-inflammatory response while impairing neutrophil trafficking," Journal of Biological Chemistry, vol. 286, no. 9, pp. 7348-7358, 2011.

[152] F. Zhang, Y. Xia, W. Yan et al., "Sphingosine 1-phosphate signaling contributes to cardiac inflammation, dysfunction, and remodeling following myocardial infarction," American Journal of Physiology Heart and Circulatory Physiology, vol. 310, no. 2, pp. H250-H261, 2016. 
[153] A. L. Franks and J. E. Slansky, "Multiple associations between a broad spectrum of autoimmune diseases, chronic inflammatory diseases and cancer," Anticancer Research, vol. 32, no. 4, pp. 1119-1136, 2012.

[154] E. Elinav, R. Nowarski, C. A. Thaiss, B. Hu, C. Jin, and R. A. Flavell, "Inflammation-induced cancer: crosstalk between tumours, immune cells and microorganisms," Nature Reviews Cancer, vol. 13, no. 11, pp. 759-771, 2013.

[155] H. Lee, J. Deng, M. Kujawski et al., "STAT3-induced S1PR1 expression is crucial for persistent STAT3 activation in tumors," Nature Medicine, vol. 16, no. 12, pp. 1421-1428, 2010.

[156] J. Liang, M. Nagahashi, E. Y. Kim et al., "Sphingosine-1phosphate links persistent STAT3 activation, chronic intestinal inflammation, and development of colitis-associated cancer," Cancer Cell, vol. 23, no. 1, pp. 107-120, 2013.

[157] S. S. Tan, L. W. Khin, L. Wong et al., "Sphingosine kinase 1 promotes malignant progression in colon cancer and independently predicts survival of patients with colon cancer by competing risk approach in South Asian population," Clinical and Translational Gastroenterology, vol. 5, no. 2, article e51, 2014.

[158] L. Abdel Hadi, C. di Vito, G. Marfia et al., "Sphingosine kinase 2 and ceramide transport as key targets of the natural flavonoid luteolin to induce apoptosis in colon cancer cells," PLoS One, vol. 10, no. 11, article e0143384, 2015.

[159] S. M. Woo, B. R. Seo, K.j. Min, and T. K. Kwon, "FTY720 enhances TRAIL-mediated apoptosis by up-regulating DR5 and down-regulating Mcl-1 in cancer cells," Oncotarget, vol. 6, no. 13, pp. 11614-11626, 2015.

[160] L. Durant, W. T. Watford, H. L. Ramos et al., "Diverse targets of the transcription factor STAT3 contribute to T cell pathogenicity and homeostasis," Immunity, vol. 32, no. 5, pp. 605-615, 2010.

[161] T. J. Harris, J. F. Grosso, H. R. Yen et al., "Cutting edge: an in vivo requirement for STAT3 signaling in TH17 development and TH17-dependent autoimmunity," Journal of Immunology, vol. 179, no. 7, pp. 4313-7, 2007.

[162] D. Iliopoulos, S. A. Jaeger, H. A. Hirsch, M. L. Bulyk, and K. Struhl, "STAT3 activation of miR-21 and miR-181b-1 via PTEN and CYLD are part of the epigenetic switch linking inflammation to cancer," Molecular Cell, vol. 39, no. 4, pp. 493-506, 2010.

[163] C. B. Williams, E. S. Yeh, and A. C. Soloff, "Tumor-associated macrophages: unwitting accomplices in breast cancer malignancy," NPJ Breast Cancer, vol. 2, no. 1, 2016.

[164] M. Aoki, H. Aoki, R. Ramanathan, N. C. Hait, and K. Takabe, "Sphingosine-1-phosphate signaling in immune cells and inflammation: roles and therapeutic potential," Mediators of Inflammation, vol. 2016, Article ID 8606878, 11 pages, 2016.

[165] S. J. Park and D. S. Im, "Sphingosine 1-phosphate receptor modulators and drug discovery," Biomolecules \& Therapeutics, vol. 25, no. 1, pp. 80-90, 2017.

[166] M. Ohtoyo, N. Machinaga, R. Inoue et al., "Component of caramel food coloring, THI, causes lymphopenia indirectly via a key metabolic intermediate," Cell Chemical Biology, vol. 23, no. 5, pp. 555-560, 2016.

[167] J. T. Bagdanoff, M. S. Donoviel, A. Nouraldeen et al., "Inhibition of sphingosine 1-phosphate lyase for the treatment of rheumatoid arthritis: discovery of (E)-1-(4-((1R,2S,3R)1,2,3,4-tetrahydroxybutyl)-1H-imidazol-2-yl)ethanone oxime (LX2931) and (1R,2S,3R)-1-(2-(isoxazol-3-yl)-1Himidazol-4-yl)butane-1,2,3,4-tetraol (LX2932)," Journal of Medicinal Chemistry, vol. 53, no. 24, pp. 8650-8662, 2010.

[168] R. Fleischmann, "Novel small-molecular therapeutics for rheumatoid arthritis," Current Opinion in Rheumatology, vol. 24, no. 3, pp. 335-341, 2012.

[169] N. Y. Hemdan, C. Weigel, C. M. Reimann, and M. H. Gräler, "Modulating sphingosine 1-phosphate signaling with DOP or FTY720 alleviates vascular and immune defects in mouse sepsis," European Journal of Immunology, vol. 46, no. 12, pp. 2767-2777, 2016.

[170] V. Fang, V. S. Chaluvadi, W. D. Ramos-Perez et al., "Gradients of the signaling lipid S1P in lymph nodes position natural killer cells and regulate their interferon- $\gamma$ response," Nature Immunology, vol. 18, no. 1, pp. 15-25, 2017.

[171] L. van der Weyden, M. J. Arends, A. D. Campbell et al., "Genome-wide in vivo screen identifies novel host regulators of metastatic colonization," Nature, vol. 541, no. 7636, pp. 233-236, 2017.

[172] M. A. Kashem, C. Wa, J. P. Wolak et al., "A high-throughput scintillation proximity assay for sphingosine-1-phosphate lyase," ASSAY and Drug Development Technologies, vol. 12, no. 5, pp. 293-302, 2014.

[173] M. A. Argiriadi, D. Banach, E. Radziejewska et al., "Creation of a S1P lyase bacterial surrogate for structure-based drug design," Bioorganic \& Medicinal Chemistry Letters, vol. 26, no. 9, pp. 2293-2296, 2016. 


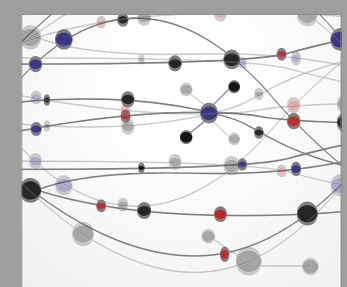

The Scientific World Journal
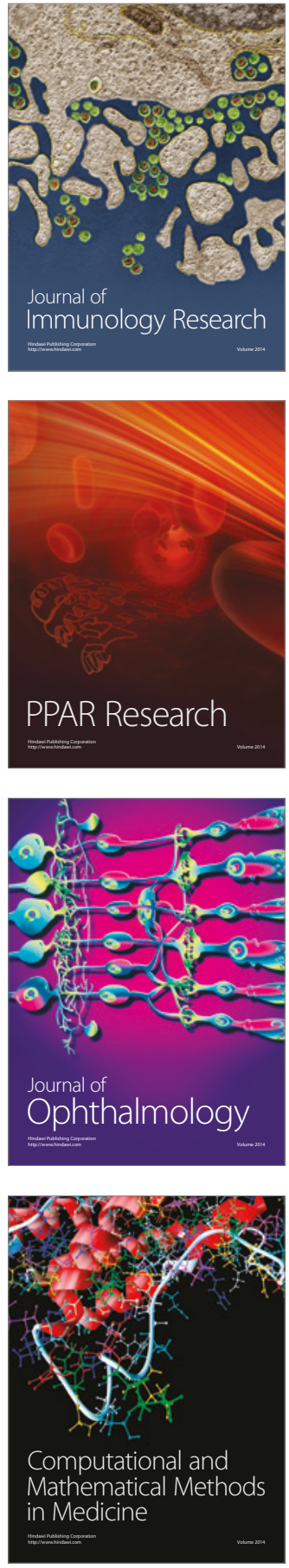

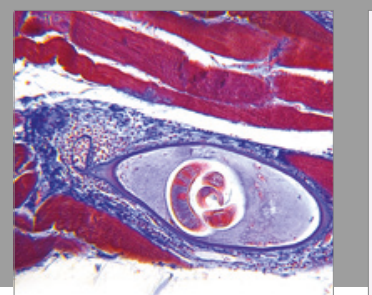

Gastroenterology Research and Practice
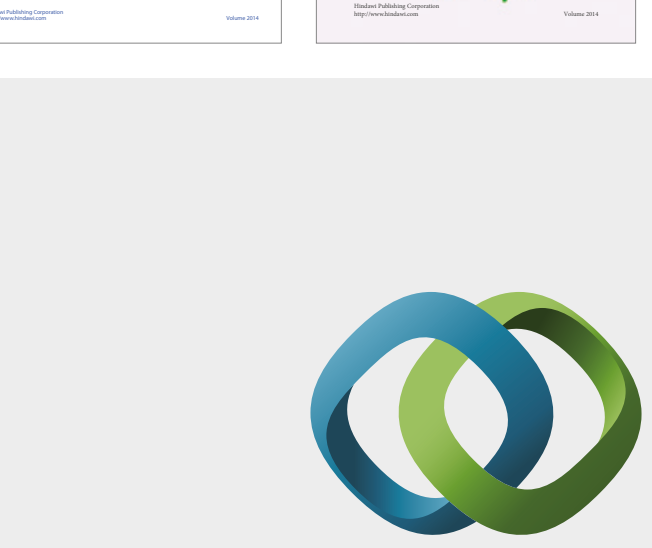

\section{Hindawi}

Submit your manuscripts at

https://www.hindawi.com
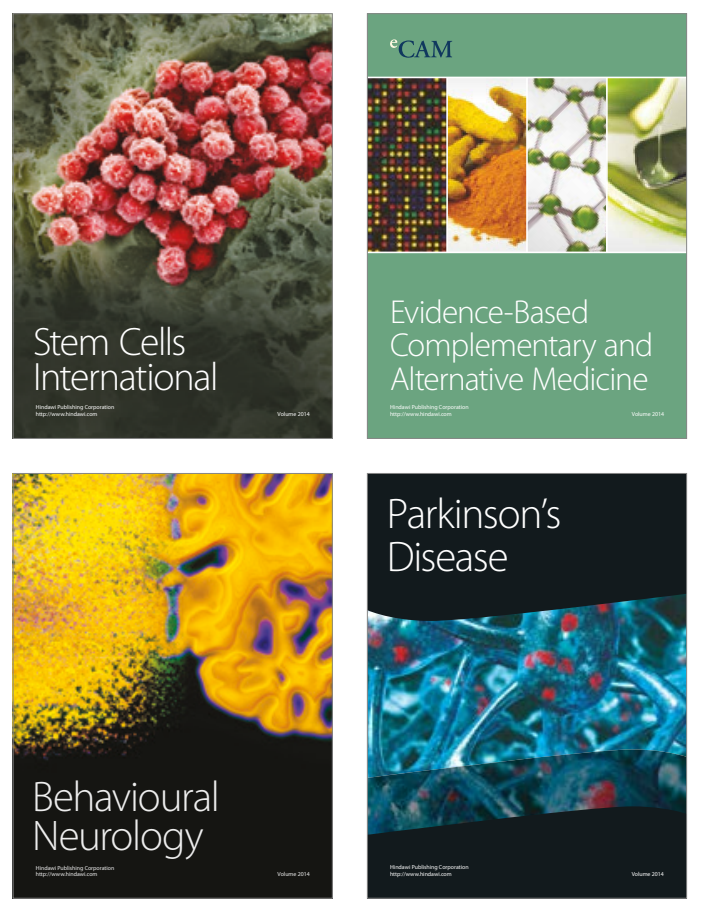
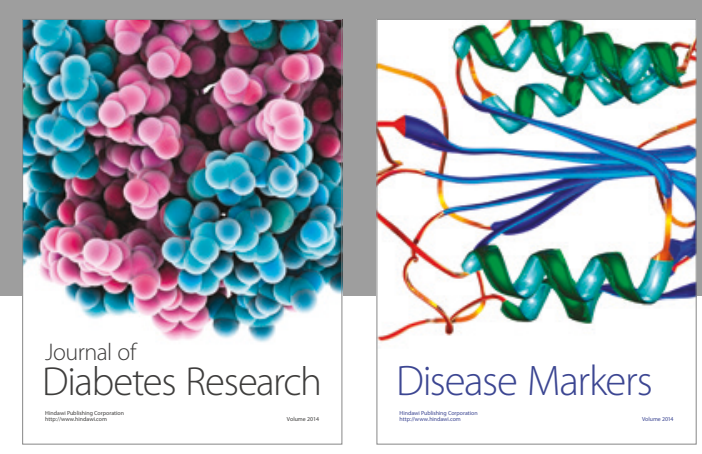

Disease Markers
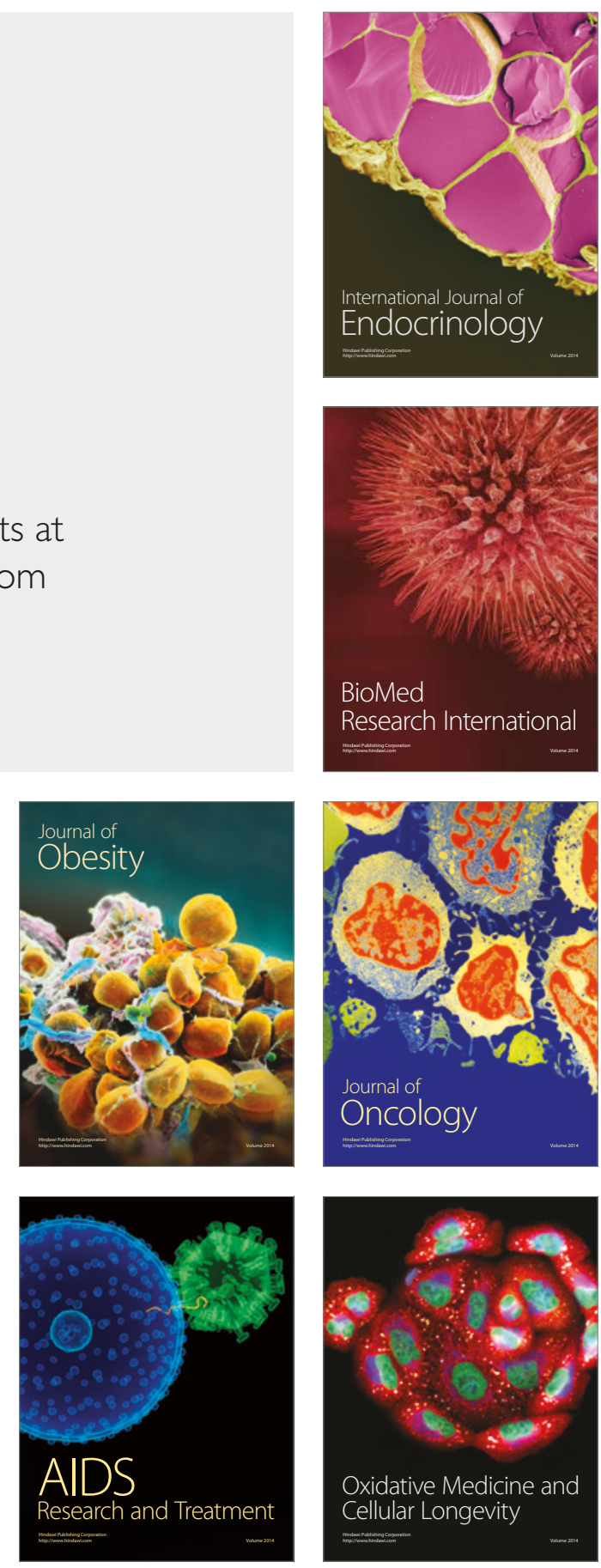